\title{
On diffusivity of a tagged particle in asymmetric zero-range dynamics
}

\author{
Sunder Sethuraman *,1 \\ 396 Carver Hall, Iowa State University, Ames, IA, 50011, USA
}

Received 13 September 2005; received in revised form 8 February 2006; accepted 3 March 2006

Available online 25 September 2006

\begin{abstract}
Consider a distinguished, or tagged particle in zero-range dynamics on $\mathbb{Z}^{d}$ with rate $g$ whose finite-range jump probabilities $p$ possess a drift $\sum j p(j) \neq 0$. We show, in equilibrium, that the variance of the tagged particle position at time $t$ is at least order $t$ in all $d \geqslant 1$, and at most order $t$ in $d=1$ and $d \geqslant 3$ for a wide class of rates $g$. Also, in $d=1$, when the jump distribution $p$ is totally asymmetric and nearest-neighbor, and the rate $g(k)$ increases, and $g(k) / k$ either decreases or increases with $k$, we show the diffusively scaled centered tagged particle position converges to a Brownian motion with a homogenized diffusion coefficient in the sense of finite-dimensional distributions. Some characterizations of the tagged particle variance are also given.

(c) 2006 Elsevier Masson SAS. All rights reserved.

\section{Résumé}

On considère «une particule marquée » pour des processus de zero-range sur $\mathbb{Z}^{d}$ avec un taux $g$ dont la probabilité de saut $p$ est à portée finie, et possède une dérive $\sum j p(j) \neq 0$. On montre qu'à l'équilibre la variance de la position de la particule marquée à l'instant $t$ pour tout $d \geqslant 1$ est au plus d'ordre $t$ si $d=1$ et $d \geqslant 3$ pour une large classe de taux $g$. Ensuite, pour le cas $d=1$, en supposant que la probabilité de saut $p$ est à plus proche voisin et totalement asymétrique, que le taux $g(k)$ est croissant, et que $g(k) / k$ est soit croissant soit décroissant avec $k$, on montre que dans le cas d'approximations par diffusion la position centrée de la particule marquée converge vers un mouvement Brownien avec des coefficients de diffusion homogènes (au sens des distributions finies). Quelques caractérisations de la variance de la particule marquée sont aussi présentées.
\end{abstract}

(c) 2006 Elsevier Masson SAS. All rights reserved.

MSC: primary $60 \mathrm{~K} 35$; secondary $60 \mathrm{~F} 05$

Keywords: Zero-range; Tagged particle; Diffusive; Variance; Invariance principle

\section{Introduction and results}

Informally, the zero-range particle system, introduced by Spitzer [35], follows the evolution of a collection of interacting random walks on $\mathbb{Z}^{d}$. Namely, from a vertex with $k$ particles, one of the particles displaces by $j$ with rate

\footnotetext{
* Tel.: +(515) 294 8140; fax: +(515) 2945454.

E-mail address: sethuram@iastate.edu (S. Sethuraman).

1 Research supported in part by NSA-H982300510041 and NSF/DMS-0504193.
} 
$(g(k) / k) p(j)$. The function on the non-negative integers $g: \mathbb{N} \rightarrow \mathbb{R}_{+}$is called the process "rate," and $p(\cdot)$ denotes the translation-invariant single particle transition probability. The above interaction is in the "time-domain," but not "spatially," hence the name "zero-range." We note the case when $g(k)$ is proportional to $k$ describes the situation of completely independent particles.

The problem of the asymptotics of a distinguished, or tagged particle interacting with others has a long history and was even mentioned in Spitzer's seminal paper. Such questions are natural and important to physics and other applications (cf. Chapters 8.I, 6.II [36]). The main difficulty in the analysis is that the tagged particle motion is not in general Markovian due to the interaction with other particles. However, the basic feeling is that in some scale the tagged particle behaves as a random walk with certain "homogenized" parameters reflecting the system dynamics. What is known in the literature, with respect to zero-range interaction, are some laws of large numbers, in equilibrium [30,32] and non-equilibrium [26], and equilibrium invariance principles when the jump probability $p$ is mean-zero, $\sum j p(j)=0[30,32]$ (see near (1.2) for exact statements of the equilibrium results).

The goal of this article is to further characterize the equilibrium fluctuations of the tagged particle when $p$ is finite-range and has a drift $\sum j p(j) \neq 0$ on which little is known. Under natural assumptions on the rate $g$, we give a characterization of the equilibrium tagged particle variance, and show it is at least diffusive in all dimensions (Theorem 1), and at most diffusive in dimension $d=1$, and under more conditions on $g$, also in $d \geqslant 3$ (Theorem 2). In addition, finite-dimensional convergence to a Brownian motion is proved in a case in $d=1$ with a "homogenized" diffusion coefficient (Theorem 3).

In contrast, we remark, with respect to a tagged particle in simple exclusion, the asymptotics are more wellstudied. In particular, laws of large numbers, both in equilibrium [29] and non-equilibrium [26] have been shown. Also, equilibrium central limit theorems and invariance principles when $p$ is mean-zero [2,27,11,37], and when $p$ has a drift in $d \geqslant 3$ [33] and in $d=1$ when $p$ is in addition nearest-neighbor [9] have been proved. See also [12,15] for fluctuations in $d=1$ with respect to a non-translation invariant $p$. Non-trivial non-equilibrium fluctuation results have even been derived in $d \geqslant 1$ when $p$ is symmetric (excluding the $d=1$ nearest-neighbor case) [25], and recently in the exceptional case in $d=1$ when $p$ is symmetric and nearest-neighbor [7]. In addition, large deviations results have been proved in some cases [24,31]. Some of these results and others are reviewed in [5], Section 4.VIII [17], Chapter 4.III [18], and Sections 4.3, 8.4 and 11.5 [10].

In this context, we note that the diffusive variance bounds for a tagged particle in zero-range given here in $d=1$ with respect to general rate $g$ and finite-range $p$ with drift (Theorem 2) have no counterpart in the simple exclusion work at the moment. Some of the techniques used in this result may have application, after suitable modification, to simple exclusion and related models.

To state assumptions and our results, we need to define more precisely the zero-range process. Let $\Sigma=\mathbb{N}^{d}$ be the configuration space where a configuration $\xi=\left\{\xi_{i}: i \in \mathbb{Z}^{d}\right\}$ is given through occupation numbers $\xi_{i}$ at vertex $i$ and $\mathbb{N}$ denotes the set of non-negative integers. The zero-range particle system then is a Markov process $\xi(t)$ on the space of right-continuous paths with left limits $D\left(\mathbb{R}_{+}, \Sigma\right)$ with formal generator defined on certain "Lipschitz" functions,

$$
(L \phi)(\xi)=\sum_{j} \sum_{i} g\left(\xi_{i}\right) p(j)\left(\phi\left(\xi^{i, i+j}\right)-\phi(\xi)\right)
$$

where $\xi^{i, i+j}$ is the configuration in which a particle from $i$ is moved to $i+j$. That is, $\xi^{i, i+j}=\xi-\delta_{i}+\delta_{i+j}$ where $\delta_{k}$ is the configuration with a single particle at $k$.

When a particle is distinguished, or tagged, we consider the joint Markov process $(x(t), \xi(t))$ on $D\left(\mathbb{R}_{+}, \mathbb{Z}^{d} \times \Sigma\right)$ where $x(t)$ is the position of the tagged particle at time $t$. The formal generator is given by

$$
\begin{aligned}
(\mathfrak{L} \psi)(x, \xi)= & \sum_{j} \sum_{i \neq x} g\left(\xi_{i}\right) p(j)\left(\psi\left(x, \xi^{i, i+j}\right)-\psi(x, \xi)\right)+\sum_{j} g\left(\xi_{x}\right) \frac{\xi_{x}-1}{\xi_{x}} p(j)\left(\psi\left(x, \xi^{x, x+j}\right)-\psi(x, \xi)\right) \\
& +\sum_{j} \frac{g\left(\xi_{x}\right)}{\xi_{x}} p(j)\left(\psi\left(x+j, \xi^{x, x+j}\right)-\psi(x, \xi)\right) .
\end{aligned}
$$

Here, the first term corresponds to particles other than at the tagged particle position $x$ moving, the second term corresponds to other particles moving from $x$, and the last term represents motion of the tagged particle itself. One can also decompose the tagged particle motion further in terms of count processes $N_{j}(t)$ which keep track of the 
number of jumps of various sizes $j$ made up to time $t$ and include their infinitesimal rates with respect to a more detailed generator.

To compensate for the non-Markov character of the tagged particle motion, a convenient method is to consider the "reference" process from the point-of-view of the tagged particle which has better properties. That is, let $\eta(t)=$ $\tau_{x(t)} \xi(t)$ where for a configuration $\omega \in \Sigma$ we define the $k$-shifted state $\tau_{k} \omega$ by $\left(\tau_{k} \omega\right)_{l}=\omega_{l+k}$ for $l \in \mathbb{Z}^{d}$. We note this "reference" process $\eta(t)$ is a Markov process, obtained from the map $\pi((x(\cdot), \xi(\cdot)))=\tau_{x(\cdot)} \xi(\cdot)$, with formal generator

$$
\begin{aligned}
(\mathcal{L} \phi)(\eta)= & \sum_{j} \sum_{i \neq 0} g\left(\eta_{i}\right) p(j)\left(\phi\left(\eta^{i, i+j}\right)-\phi(\eta)\right)+\sum_{j} g\left(\eta_{0}\right) \frac{\eta_{0}-1}{\eta_{0}} p(j)\left(\phi\left(\eta^{0, j}\right)-\phi(\eta)\right) \\
& +\sum_{j} \frac{g\left(\eta_{0}\right)}{\eta_{0}} p(j)\left(\phi\left(\tau_{j}\left(\eta^{0, j}\right)\right)-\phi(\eta)\right) .
\end{aligned}
$$

In words, $\tau_{j}\left(\eta^{0, j}\right)$ is the configuration obtained by displacing the tagged particle by $j$ and then shifting accordingly the reference frame.

The construction of these systems requires some conditions on $g$ and $p$.

Basic assumptions on $g$ and $p$. We will assume throughout $g(0)=0, g(k)>0$ for $k \geqslant 1,|g(k+1)-g(k)| \leqslant K$ for some constant $K$, and $\liminf _{k \rightarrow \infty} g(k)>0$, and also $p(0)=0$ and $p$ is finite-range, that is $p(i)=0$ for $|i|>R$ for some $1 \leqslant R<\infty$, whose symmetrization $s(x)=(p(x)+p(-x)) / 2$ is irreducible.

We remark of these assumptions, the condition liminf $g(k)>0$ is made to ensure invariant measures exist (cf. [1]). When violated, the system evolution can lead to aggregation of particles in large piles, a sort of "condensation" effect (cf. $[8,6])$.

Under weaker assumptions, which include the above, Andjel constructs the process $\xi(t)$ semigroup $T_{t}^{L}$ and generator $L$ on a class of "Lipschitz" functions $\mathcal{D}^{\prime}$ defined on a subset $\Sigma^{\prime} \subset \Sigma$ of the configuration space,

$$
\begin{aligned}
& \Sigma^{\prime}=\left\{\xi:\|\xi\|=\sum_{i \in \mathbb{Z}^{d}}\left|\xi_{i}\right| \beta_{i}<\infty\right\} \\
& \mathcal{D}^{\prime}=\left\{f:\left|f\left(\xi^{\prime}\right)-f\left(\xi^{\prime \prime}\right)\right| \leqslant c\left\|\xi^{\prime}-\xi^{\prime \prime}\right\| \text { for all } \xi^{\prime}, \xi^{\prime \prime} \in \Sigma^{\prime}, \text { for some } c=c(f)\right\}
\end{aligned}
$$

where one can take $\beta_{i}=\sum_{n \geqslant 0} 2^{-n} s^{(n)}(i)$ for instance [1]. In a similar way, one can construct the process $(x(t), \xi(t))$ semigroup $T_{t}^{\mathfrak{L}}$ and generator $\mathfrak{L}$ with respect to "Lipschitz" functions $f$ where

$$
\left|f\left(\left(x, \xi^{\prime}\right)\right)-f\left(\left(y, \xi^{\prime \prime}\right)\right)\right| \leqslant c\left[|x-y|+\left\|\xi^{\prime}-\xi^{\prime \prime}\right\|\right]
$$

for all $x, y \in \mathbb{Z}^{d}, \xi^{\prime}, \xi^{\prime \prime} \in \Sigma^{\prime}$ with $\xi_{x}^{\prime}, \xi_{y}^{\prime \prime} \geqslant 1$ for some $c=c(f)$. Then, from the map $\pi$, process $\eta(t)$ semigroup $T_{t}^{\mathcal{L}}$ and generator $\mathcal{L}$ can be constructed on $\mathcal{D}$, the space of "Lipschitz" functions on $\Sigma^{\prime \prime}=\left\{\xi \in \Sigma^{\prime}: \xi_{0} \geqslant 1\right\}$, namely those $f$ so that $\left|f\left(\xi^{\prime}\right)-f\left(\xi^{\prime \prime}\right)\right| \leqslant c\left\|\xi^{\prime}-\xi^{\prime \prime}\right\|$ for all $\xi^{\prime}, \xi^{\prime \prime} \in \Sigma^{\prime \prime}$ for some $c=c(f)$.

The zero-range process $\xi(t)$ has a well-known explicit family product invariant measures $R_{\alpha}=\prod_{i \in \mathbb{Z}^{d}} \mu_{\alpha}$ for $0 \leqslant \alpha<\liminf g(k)$ with marginal supported on non-negative integers,

$$
\mu_{\alpha}(k)=\frac{1}{Z_{\alpha}} \frac{\alpha^{k}}{g(k) !} \quad \text { for } k \geqslant 1 \quad \text { and } \quad \mu_{\alpha}(0)=\frac{1}{Z_{\alpha}}
$$

where $g(k) !=g(1) \cdots g(k)$ and $Z_{\alpha}$ is the normalization [1]. These measures are all supported on $\Sigma^{\prime}$, and it can be shown that the process begun on $\Sigma^{\prime}$ never leaves $\Sigma^{\prime}$. Also, it is not difficult to see that $\mathcal{D}^{\prime}$ is a dense subset of $L^{2}\left(R_{\alpha}\right)$. Let now $\rho(\alpha)=\sum_{k} k \mu_{\alpha}(k)$, be the mean of the marginal $\mu_{\alpha}$, that is, the "density" of particles under $R_{\alpha}$, and let $\rho^{*}=\lim _{\alpha \uparrow \liminf g(k)} \rho(\alpha)$. Note that $\rho^{*}$ may be finite for some type of $g$ 's. As $\rho(\alpha) \uparrow \rho^{*}$ for $\alpha \uparrow \liminf g(k)$, for a given $0 \leqslant \rho<\rho^{*}$, there is a unique inverse $\alpha=\alpha(\rho)$.

For the reference process $\eta(t)$, the "Palm" or origin size biased measures given by $\mathrm{d} Q_{\alpha}=\left(\eta_{0} / \rho(\alpha)\right) \mathrm{d} R_{\alpha}$ are invariant (cf. [23,30]). Only the marginal at the origin, supported on the positive integers and denoted by $\mu_{\alpha}^{0}$, differs from $\mu_{\alpha}$, namely

$$
\mu_{\alpha}^{0}(k)=\frac{1}{\rho Z_{\alpha}} \frac{k \alpha^{k}}{g(k) !} \quad \text { for } k \geqslant 1 \quad \text { and } \quad \mu_{\alpha}^{0}(k)=0 \quad \text { otherwise. }
$$


Analogous to the discussion with respect to $\xi(t)$ and $R_{\alpha}$, the measure $Q_{\alpha}$ is supported on $\Sigma^{\prime \prime}$, and it can be shown that the reference process $\eta(t)$ begun on $\Sigma^{\prime \prime}$ never leaves $\Sigma^{\prime \prime}$. Also, $\mathcal{D}$ is a dense subset of $L^{2}\left(Q_{\alpha}\right)$.

We now comment that, with respect to an invariant $R_{\alpha}$, one can extend the zero-range process semigroup $T_{t}^{L}$ and generator $L$ to a strongly continuous semigroup and closed generator with respect to $L^{2}\left(R_{\alpha}\right)$ so that bounded functions in $\mathcal{D}^{\prime}$ form a core of the domain of $L$; in fact, the same arguments show that $\mathcal{D}^{\prime}$ itself is a core (cf. Section 2 [32]; for general reference see Chapter 1 [4]). In the same way, with respect to a $Q_{\alpha}$, the reference semigroup $T_{t}^{\mathcal{L}}$ and generator $\mathcal{L}$ can be extended to a strongly continuous semigroup and closed generator on $L^{2}\left(Q_{\alpha}\right)$ with core $\mathcal{D}$ for the domain of $\mathcal{L}$ denoted $\mathbb{D}_{\alpha}$. We note also here constructions of these processes can be made through the martingale-problem approach $[30,28]$. Also, in this context, we note a Hille-Yosida type approach [16].

In addition, we note both families $\left\{R_{\alpha}\right\}$ and $\left\{Q_{\alpha}\right\}$ are in fact extremal measures in their respective convex set of invariant measures, and so process evolutions starting from these invariant states are time shift-ergodic. Extremality of $R_{\alpha}$ is proved in [32]; analogous arguments apply to $Q_{\alpha}$.

Also, we note a standard computation shows that the adjoint $\mathcal{L}^{*}$ with respect to $Q_{\alpha}$ is itself a reference process generator but with reversed jump probability $p^{*}(\cdot)=p(-\cdot)$. Hence, in equilibrium $Q_{\alpha}$, the time-reversed process at time $t,\{\eta(t-u): 0 \leqslant u \leqslant t\}$, is also a reference process in equilibrium $Q_{\alpha}$ but with respect to jump probability $p^{*}$.

In the following, to avoid degeneracies, we will work with a fixed $0<\alpha<\liminf g(k)$ for which $\rho=\rho(\alpha)>0$, and corresponding $R_{\alpha}$ and $Q_{\alpha}$. For simplicity, we denote by $E_{\alpha}[\cdot]$ the expectation under $Q_{\alpha}$ and also under the reference process measure starting from $Q_{\alpha}$ when there is no confusion; otherwise, the underlying measure is noted as a suffix.

Write now the tagged particle position $x(t)$ at time $t$ as the sum total displacement, that is,

$$
x(t)=\sum_{j} j N_{j}(t)
$$

where $N_{j}(t)$ is the number of jumps of size $j$ it makes, or in terms of the reference process, the count of size $j$-shifts made up to time $t$. The count $N_{j}(t)$ is compensated by $\int_{0}^{t}\left(g\left(\eta_{0}(s) / \eta_{0}(s)\right) p(j) \mathrm{d} s\right.$, so that further

$$
x(t)=\sum_{j} j M_{j}(t)+\sum_{j} j \int_{0}^{t} \frac{g\left(\eta_{0}(s)\right)}{\eta_{0}(s)} p(j) \mathrm{d} s
$$

where $M_{j}(t)=N_{j}(t)-\int_{0}^{t}\left(g\left(\eta_{0}(s) / \eta_{0}(s)\right) p(j) \mathrm{d} s\right.$, since jumps of different sizes cannot happen simultaneously, are orthogonal martingales for $j \in \mathbb{Z}^{d}$. Moreover, we note $M_{j}^{2}(t)-\int_{0}^{t}\left(g\left(\eta_{0}(s)\right) / \eta_{0}(s)\right) p(j) \mathrm{d} s$ are also martingales for $j \in \mathbb{Z}^{d}$.

So, the tagged position $x(t)$ can be thought of as a function of the reference process. For most of the paper, we will think in terms of the reference frame, that is, the notation $x(t)$ will denote the total reference shift $\sum j N_{j}(t)$. It will also be useful to define

$$
M(t)=\sum_{j} j M_{j}(t) \quad \text { and } \quad A(t)=\int_{0}^{t} \mathfrak{f}(\eta(s)) \mathrm{d} s
$$

where $\mathfrak{f}(\eta)=\left(\sum j p(j)\right)\left[g\left(\eta_{0}\right) / \eta_{0}-\alpha / \rho\right]$. Note, in equilibrium $Q_{\alpha}$, one obtains, as $E_{\alpha}\left[g\left(\eta_{0}\right) / \eta_{0}\right]=\alpha / \rho$, that

$$
E_{\alpha}[x(t)]=t E_{\alpha}\left[\frac{g\left(\eta_{0}\right)}{\eta_{0}}\right] \sum_{j} j p(j)=t \frac{\alpha}{\rho} \sum_{j} j p(j)
$$

and so $x(t)-E_{\alpha}[x(t)]=M(t)+A(t)$. Also, from orthogonality of the $M_{j}$ 's, $E_{\alpha}\left[|M(t)|^{2}\right]=t(\alpha / \rho) \sum|j|^{2} p(j)$.

Before going to the main results, we briefly review the known zero-range equilibrium tagged particle asymptotics. In equilibrium $Q_{\alpha}$, the law of large numbers holds [30,32]:

$$
\lim _{t \rightarrow \infty} \frac{1}{t} x(t)=\frac{\alpha}{\rho} \sum_{j} j p(j) \quad \text { a.s. }
$$


Also, with respect to fluctuations, when the jump probabilities are mean-zero, $\sum j p(j)=0$, then $x(t)=\sum j M_{j}(t)$ is a martingale as the compensator terms cancel in (1.1). Then, under equilibrium $Q_{\alpha}$, the quadratic variation is

$$
\frac{E_{\alpha}\left[|x(t)|^{2}\right]}{t}=\frac{1}{t} \sum_{j}|j|^{2} E_{\alpha}\left[\int_{0}^{t} \frac{g\left(\eta_{0}(s)\right)}{\eta_{0}(s)} p(j) \mathrm{d} s\right]=\frac{\alpha}{\rho} \sum_{j}|j|^{2} p(j)
$$

and by martingale central limit theorem the invariance principle is proved [30,32]:

$$
\frac{1}{\sqrt{\lambda}} x(\lambda t) \Rightarrow \mathbb{B}(t) \quad \text { as } \lambda \rightarrow \infty
$$

where $\mathbb{B}(t)$ is $d$-dimensional Brownian motion with covariance matrix $\left[(\alpha / \rho) t \sum_{j}\left(e_{i} \cdot j\right)\left(e_{k} \cdot j\right) p(j)\right]$ in terms of the standard basis $\left\{e_{i}\right\}$ of $\mathbb{Z}^{d}$.

We now arrive at our first result which gives a characterization of the tagged particle variance, and states that it is at least diffusive in all dimensions. As a comparison, we note this is not true for simple exclusion, in the case $d=1$ and the jump probability $p$ is nearest-neighbor symmetric, for which the variance at time $t$ is order $t^{1 / 2}$ [2]. See also $[3,14]$ for variance representations with respect to exclusion processes.

Define the measure $\mu_{\alpha}^{\prime}$ supported on the positive integers by

$$
\mu_{\alpha}^{\prime}(k)=\frac{1}{Z_{\alpha}^{\prime}} \frac{\alpha^{k-1}}{g(k-1) !} \quad \text { for } k \geqslant 2 \quad \text { and } \quad \mu_{\alpha}^{\prime}(1)=\frac{1}{Z_{\alpha}^{\prime}}
$$

with normalization $Z_{\alpha}^{\prime}$. The interpretation is that $\mu_{\alpha}^{\prime}$ puts a particle at the origin and distributes other particles there according to $\mu_{\alpha}$. Let $Q_{\alpha}^{\prime}=\prod_{i \neq 0} \mu_{\alpha} \times \mu_{\alpha}^{\prime}$ and let $E_{\alpha}^{\prime}$ denote expectation under the reference process begun at $Q_{\alpha}^{\prime}$.

Theorem 1. Under initial distribution $Q_{\alpha}$, we have in all dimensions $d \geqslant 1$ for $t \geqslant 0$ that

$$
E_{\alpha}\left[\left|x(t)-E_{\alpha}[x(t)]\right|^{2}\right]=\frac{\alpha}{\rho} \sum_{j}|j|^{2} p(j) t+E_{\alpha}\left[|A(t)|^{2}\right] \geqslant \frac{\alpha}{\rho} \sum_{j}|j|^{2} p(j) t .
$$

The lower bound is strict unless $g(k)$ is proportional to $k$. Also, the term $E_{\alpha}\left[|A(t)|^{2}\right]$ is further evaluated as

$$
E_{\alpha}\left[|A(t)|^{2}\right]=\frac{\alpha}{\rho}\left[\sum_{j} j p(j)\right] \cdot 2 \int_{0}^{t}\left\{E_{\alpha}^{\prime}[x(s)]-E_{\alpha}[x(s)]\right\} \mathrm{d} s .
$$

The first term in (1.3), $(\alpha / \rho) \sum|j|^{2} p(j) t$, is the mean quadratic variation of the martingale $M(t)$, and can be thought of as a "dynamical" contribution to the tagged particle variance. The second term $E_{\alpha}\left[|A(t)|^{2}\right]$, on the other hand, from (1.4), as a difference in expected tagged particle positions from different initial measures, represents in a sense variation due to initial conditions. We also note when $g(k)=c k$ is proportional to $k$, the case of independent particles, that $\alpha / \rho=c, Q_{\alpha}^{\prime}=Q_{\alpha}$ and $\mathfrak{f} \equiv 0$, and so the tagged particle variance reduces to the variance of a single random walk with jump rates $c p$.

Now, to give upperbounds on the tagged particle variance, we describe a class of rate functions $g$.

Assumption (SP). Let $L_{n}$ be the generator of the symmetric zero-range process on a cube $B_{n}=\left\{i \in \mathbb{Z}^{d}\right.$ : $\left.\max _{l}\left(\left|i_{l}\right|\right) \leqslant n\right\}$, namely $\left(L_{n} \phi\right)(\xi)=\sum_{i, j \in B_{n}} g\left(\xi_{i}\right)\left(\phi\left(\xi^{i, j}\right)-\phi(\xi)\right) s(j-i)$. Let $W(n, M)$ be the inverse of the spectral gap of $L_{n}$ when there are $M$ particles in $B_{n}$. Then, we assume the rate $g$ is such that there is a constant $C=C(\alpha, g, p, d)$ where $E_{R_{\alpha}}\left[\left(W\left(n, \sum_{i \in B_{n}} \xi_{i}\right)\right)^{2}\right] \leqslant C n^{4}$.

We observe rates $g$ where $W(n, M) \leqslant C n^{2}$ for a constant $C$ independent of $M$, satisfy (SP) trivially, and include those rates where, for some $a \geqslant 1$ and $b>0, g(k+a)-g(k) \geqslant b$ for all $k \geqslant 0$ [13]. Also, for the rate $g(k)=1_{[k \geqslant 1]}$, it is known $W(n, M) \leqslant C(1+M / n)^{2} n^{2}$ for some constant $C$ [19], and so (SP) holds. It is most likely true that all rates $g$ satisfy (SP), although this is open. 
Theorem 2. Under initial distribution $Q_{\alpha}$, when $\sum j p(j) \neq 0$, we have in $d=1$ (without further assumptions), and in $d \geqslant 3$ under Assumption (SP) that there is a constant $C=C(\alpha, g, p, d)$ where, for $t \geqslant 0$,

$$
E_{\alpha}\left[\left|x(t)-E_{\alpha}[x(t)]\right|^{2}\right] \leqslant C t .
$$

We note, unfortunately, our estimates made in $d=1$ and $d \geqslant 3$ do not seem to carry over straightforwardly to dimension $d=2$. An open question then is to investigate the tagged particle variance in $d=2$ which should also be diffusive.

We now state finite-dimensional convergence to a Brownian motion in a special case in $d=1$.

Assumption (ID $\downarrow$ ). The rate function $g$ is such that $g(k)$ increases and $g(k) / k$ decreases with $k$.

Assumption (ID $\uparrow$ ). The rate function $g$ is such that both $g(k)$ and $g(k) / k$ increase with $k$.

Examples under (ID $\downarrow$ ) include the well-studied case $g(k)=1_{[k \geqslant 1]}$. We note also, under (ID $\uparrow$ ), and our basic assumptions (which preclude "super-linearity"), $g(k) / k$ increases to a bounded limit.

Theorem 3. Under initial distribution $Q_{\alpha}$, in $d=1$ when the jump probability is totally asymmetric $p(1)=1$ and $g$ satisfies either Assumption (ID $\downarrow$ ) or (ID $\uparrow$ ), we have the tagged particle variance $V(t)=E_{\alpha}\left[\left(x(t)-E_{\alpha}[x(t)]\right)^{2}\right]$ is super-additive in $t \geqslant 0$, and also finite-dimensional convergence

$$
\lim _{\lambda \rightarrow \infty} \frac{1}{\sqrt{\lambda}}\left(x(\lambda t)-E_{\alpha}[x(\lambda t)]\right)=\mathbb{B}(t)
$$

to a Brownian motion $\mathbb{B}$ with diffusion coefficient $\sigma^{2}=\sigma^{2}(\alpha, g)$ satisfying

$$
C \geqslant \sigma^{2}=\frac{\alpha}{\rho}\left[1+\sup _{t>0} \frac{2}{t} \int_{0}^{t}\left\{E_{\alpha}^{\prime}[x(s)]-E_{\alpha}[x(s)]\right\} \mathrm{d} s\right] \geqslant \frac{\alpha}{\rho}
$$

where $C$ is the constant from Theorem 2. Also, the lower bound is strict unless $g(k)$ is proportional to $k$.

We note an ingredient in the proof of Theorem 3 is to show the corresponding tagged particle position has weakly positively correlated increments (Lemma 4.2). In comparison, however, a tagged particle in $d=1$ simple exclusion with totally asymmetric nearest-neighbor transitions has negatively correlated increments, and in fact is a Poisson process (cf. Section 4.VIII [17]). But, in the above zero-range context, exactly when the system is that of independent particles (when $g(k)$ is proportional to $k$ ) is the tagged particle a Poisson process, as otherwise $\sigma^{2}(\rho)>\alpha / \rho=$ $E_{\alpha}[x(1)]$.

Also, we note a natural open question is to show tightness of the scaled centered positions in $D[0, \infty)$.

Remark on extensions. Presumably, one should expect diffusive variance bounds, and corresponding central limit theorems and invariance principles on the tagged position under only our initial basic assumptions on $g$ and $p$ in all dimensions not just the cases considered here. Also, in principle, one should expect the "finite-range" condition on $p$ could be relaxed to a "second-moment" condition with analogous results. Finally, what possible different behaviors might arise under further weakening of the basic assumptions would be of interest and have not been considered.

We now comment on the proofs of these results, and the plan of the paper. The proof of Theorem 1, in Section 2, follows from a short argument using time-reversal. The proof of Theorem 2, which forms the bulk of the paper, follows from an analysis of certain resolvent or $H_{-1}$ norms, and is found in Section 3. On a technical level, we note the application of $H_{-1}$ norm estimates in the asymmetric zero-range context differs somewhat from previous simple exclusion methods (cf. [33]) which use concepts of "dual" basis functions to reduce calculations. Last, the proof of Theorem 3, in Section 4, makes use of the diffusive variance bounds in Theorem 2, and follows by applying a Newman-Wright theorem. These proofs can be read independently of each other. 


\section{Proof of Theorem 1}

Most of the proof follows from explicit calculations. We have

$$
\begin{aligned}
E_{\alpha}\left[\left|x(t)-E_{\alpha}[x(t)]\right|^{2}\right] & =E_{\alpha}\left[|M(t)+A(t)|^{2}\right] \\
& =E_{\alpha}\left[|M(t)|^{2}\right]+2 E_{\alpha}[M(t) \cdot A(t)]+E_{\alpha}\left[|A(t)|^{2}\right] \\
& =\frac{\alpha}{\rho} \sum|j|^{2} p(j) t+2 \int_{0}^{t} E_{\alpha}[M(s) \cdot \mathfrak{f}(\eta(s))] \mathrm{d} s+E_{\alpha}\left[|A(t)|^{2}\right] .
\end{aligned}
$$

Now, as noted in the introduction, under time reversal at time $s$, with respect to the process begun under $Q_{\alpha}$, $\left\{\eta^{*}(u)=\eta(s-u): 0 \leqslant u \leqslant s\right\}$ is a reference frame zero-range process in equilibrium $Q_{\alpha}$ with reversed rates $p(-\cdot)$. Note also that the number of $j$-shifts up to time $s$ in the forward realization equals the number of $-j$-shifts in the timereversed realization up to time $s$, that is $N_{j}(s ; \eta(\cdot))=N_{-j}\left(s ; \eta^{*}(\cdot)\right)$ where denoted in each count is the directed realization to which it refers. Also, in this notation, $M^{*}(s ; \eta(\cdot))=\sum j N_{-j}(s ; \eta(\cdot))-\sum j \int_{0}^{s}\left(g\left(\eta_{0}(u)\right) / \eta_{0}(u)\right) p(j) \mathrm{d} u$ is a martingale with respect to the reversed process with reversed rates $p(-\cdot)$.

So, we have

$$
\begin{aligned}
E_{\alpha}[M(s) \cdot \mathfrak{f}(\eta(s))] & =E_{\alpha}\left[M^{*}\left(s ; \eta^{*}(\cdot)\right) \cdot \mathfrak{f}\left(\eta^{*}(0)\right)\right]=E_{\alpha}^{*}\left[M^{*}(s ; \eta(\cdot)) \cdot \mathfrak{f}(\eta(0))\right] \\
& =\int E_{\eta}^{*}\left[M^{*}(s ; \eta(\cdot))\right] \cdot \mathfrak{f}(\eta) \mathrm{d} Q_{\alpha}(\eta)
\end{aligned}
$$

after conditioning on time 0 where $E_{\alpha}^{*}$ and $E_{\eta}^{*}$ denote expectations with respect to the reversed process with initial distribution $Q_{\alpha}$ and initial state $\eta$ respectively. As $E_{\eta}^{*}\left[M^{*}(s ; \eta(\cdot)]=0\right.$, we have $E_{\alpha}[M(s) \cdot \mathfrak{f}(\eta(s))]=0$, and substituting into (2.1) we obtain (1.3).

To derive (1.4), we write using stationarity that

$$
\begin{aligned}
E_{\alpha}\left[|A(t)|^{2}\right] & =2 \int_{0}^{t} E_{\alpha}\left[\mathfrak{f}(\eta(0)) \cdot \int_{0}^{s} \mathfrak{f}(\eta(u)) \mathrm{d} u\right] \mathrm{d} s=2 \int_{0}^{t} E_{\alpha}[\mathfrak{f}(\eta(0)) \cdot A(s)] \mathrm{d} s \\
& =2 \int_{0}^{t} E_{\alpha}\left[\mathfrak{f}(\eta(0)) \cdot\left(\left(x(s)-E_{\alpha}[x(s)]\right)-M(s)\right)\right] \mathrm{d} s=2 \int_{0}^{t} E_{\alpha}[\mathfrak{f}(\eta(0)) \cdot x(s)] \mathrm{d} s
\end{aligned}
$$

as $\mathfrak{f}$ is mean-zero, and $E_{\alpha}[\mathfrak{f}(\eta(0)) \cdot M(s)]=E_{\alpha}\left[\mathfrak{f}(\eta(0)) \cdot E_{\eta(0)}[M(s)]\right]=0$.

Now, by a simple calculation, for a function $h \in L^{2}\left(Q_{\alpha}\right)$, we have

$$
E_{\alpha}\left[\left(g\left(\eta_{0}\right) / \eta_{0}\right) h(\eta)\right]=\frac{\alpha}{\rho} E_{R_{\alpha}}\left[E_{\eta+\delta_{0}}[h(\eta)]\right]=\frac{\alpha}{\rho} E_{Q_{\alpha}^{\prime}}[h(\eta)] .
$$

Hence, noting the definition of $\mathfrak{f}$ (cf. near (1.1)),

$$
E_{\alpha}[\mathfrak{f}(\eta(0)) \cdot x(s)]=E_{\alpha}\left[\mathfrak{f}(\eta(0)) \cdot E_{\eta(0)}[x(s)]\right]=\frac{\alpha}{\rho}\left(\sum j p(j)\right) \cdot\left\{E_{\alpha}^{\prime}[x(s)]-E_{\alpha}[x(s)]\right\} .
$$

We now consider when $E_{\alpha}\left[|A(t)|^{2}\right]$ is positive. When $g(k)$ is proportional to $k$, as noted in the remark after the Theorem 1 statement, $\mathfrak{f} \equiv 0$, and so $E_{\alpha}\left[|A(t)|^{2}\right]=0$ for all $t \geqslant 0$ in this case. When $g(k)$ is not linear in $k, \mathfrak{f}$ is nontrivial, and $\lim _{s \downarrow 0} s^{-2} E_{\alpha}\left[|A(s)|^{2}\right]=E_{\alpha}\left[|\mathfrak{f}|^{2}\right]>0$. Hence, in this case, $E_{\alpha}\left[|A(t)|^{2}\right]>t^{2} E_{\alpha}\left[|\mathfrak{f}|^{2}\right] / 2>0$ for all $t>0$ small. Now, suppose $E_{\alpha}\left[\left|A\left(t_{0}\right)\right|^{2}\right]=0$ for some $t_{0}>0$. Then, $\int_{0}^{t_{0}} \mathfrak{f}(\eta(s)) \mathrm{d} s=0$ a.s. By stationarity, $\int_{a}^{t_{0}+a} \mathfrak{f}(\eta(s)) \mathrm{d} s=$ 0 a.s. for each $a>0$. By simple addition and subtraction, for $0<\epsilon<t_{0}$, we have $\int_{0}^{\epsilon} \mathfrak{f}(\eta(s)) \mathrm{d} s=\int_{k t_{0}}^{k t t_{0}+\epsilon} \mathfrak{f}(\eta(s)) \mathrm{d} s$ a.s. for each integer $k \geqslant 1$. Then,

$$
\int_{0}^{\epsilon} \mathfrak{f}(\eta(s)) \mathrm{d} s=\frac{1}{n} \sum_{k=1}^{n} \int_{k t_{0}}^{k t_{0}+\epsilon} \mathfrak{f}(\eta(s)) \mathrm{d} s \quad \text { a.s. }
$$




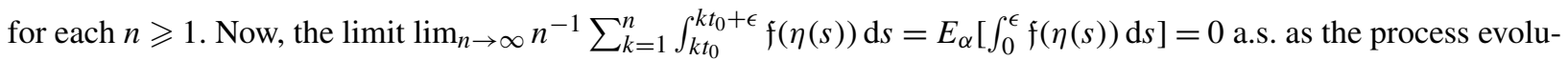
tion starting from $Q_{\alpha}$ is time-shift ergodic, as noted in the introduction. Hence, $\int_{0}^{\epsilon} \mathfrak{f}(\eta(s)) \mathrm{d} s=0$ a.s. Choosing now $\epsilon>0$ small enough so that $E_{\alpha}\left[|A(\epsilon)|^{2}\right]>0$ however yields a contradiction, finishing the proof.

\section{Proof of Theorem 2}

We first discuss some definitions and estimates involving variational formulas for some resolvent quantities involving notation $E_{\alpha}[f \psi]=\langle f, \psi\rangle_{\alpha}$, and $E_{\alpha}\left[f^{2}\right]=\|f\|_{0}^{2}$.

The generator $\mathcal{L}$, with respect to $Q_{\alpha}$, can be decomposed into symmetric and anti-symmetric parts, $\mathcal{L}=\mathcal{S}+\mathcal{A}$ where $\mathcal{S}=\left(\mathcal{L}+\mathcal{L}^{*}\right) / 2$ and $\mathcal{A}=\left(\mathcal{L}-\mathcal{L}^{*}\right) / 2$ are well defined on $\mathcal{D}$. One can check that the symmetric operator $\mathcal{S}$ is in fact the generator of the reference frame zero-range process with symmetrized jump probabilities $s(\cdot)$. Moreover, $-\mathcal{S}$ is a non-negative operator whose Dirichlet form can be computed, for $\psi \in \mathcal{D}$, as

$$
\begin{aligned}
\langle\psi,(-\mathcal{S}) \psi\rangle_{\alpha}= & \frac{1}{2} \sum_{j} \sum_{i \neq 0} E_{\alpha}\left[g\left(\eta_{i}\right)\left(\psi\left(\eta^{i, i+j}\right)-\psi(\eta)\right)^{2}\right] s(j) \\
& +\frac{1}{2} \sum_{j} E_{\alpha}\left[g\left(\eta_{0}\right) \frac{\eta_{0}-1}{\eta_{0}}\left(\psi\left(\eta^{0, j}\right)-\psi(\eta)\right)^{2}\right] s(j) \\
& +\frac{1}{2} \sum_{j} E_{\alpha}\left[\frac{g\left(\eta_{0}\right)}{\eta_{0}}\left(\psi\left(\tau_{j}\left(\eta^{0, j}\right)\right)-\psi(\eta)\right)^{2}\right] s(j) .
\end{aligned}
$$

We now consider some useful Hilbert spaces. For $\lambda>0$ and $\psi \in \mathcal{D}$, define the norm $\|\psi\|_{1, \lambda}$ by

$$
\|\psi\|_{1, \lambda}^{2}=\langle\psi,(\lambda-\mathcal{S}) \psi\rangle_{\alpha} .
$$

The Hilbert space $H_{1, \lambda}$ then is the completion over $\mathcal{D}$ with respect to this norm.

To define a dual norm, consider, for $f \in L^{2}\left(Q_{\alpha}\right)$ and $\psi \in \mathcal{D}$, that $\langle f, \psi\rangle_{\alpha} \leqslant\|f\|_{0}\|\psi\|_{0} \leqslant \lambda^{-1 / 2}\|f\|_{0}\|\psi\|_{1, \lambda}$. Hence, for $f \in L^{2}\left(Q_{\alpha}\right)$, the dual norm

$$
\|f\|_{-1, \lambda}=\inf \left\{\kappa:\langle f, \psi\rangle_{\alpha} \leqslant \kappa\|\psi\|_{1, \lambda} \text { for all } \psi \in \mathcal{D}\right\}
$$

is always finite with bound $\|f\|_{-1, \lambda}^{2} \leqslant \lambda^{-1}\|f\|_{0}^{2}$. In particular, for $\psi \in \mathcal{D}$, we have

$$
\langle f, \psi\rangle_{\alpha} \leqslant\|f\|_{-1, \lambda}\|\psi\|_{1, \lambda} \leqslant\left[\frac{1}{\lambda}\|f\|_{0}^{2}\right]^{1 / 2}\|\psi\|_{1, \lambda} .
$$

Define, correspondingly, $H_{-1, \lambda}$ as the Hilbert space with respect to the norm $\|\cdot\|_{-1, \lambda}$. [Although we will not need it, we remark that $\|f\|_{-1, \lambda}$ can be evaluated as $\|f\|_{-1, \lambda}^{2}=\left\langle f,(\lambda-\mathcal{S})^{-1} f\right\rangle_{\alpha}$; see also [22] for other contexts.]

It will also be convenient to define (semi-)norm $\|\psi\|_{1}$ for $\psi \in \mathcal{D}$ by $\|\psi\|_{1}^{2}=\langle\psi,(-S) \psi\rangle_{\alpha}$, and corresponding Hilbert space $H_{1}$ as the completion over $\mathcal{D}$ with respect to this norm, after modding out by zero-norm functions. Also, for $f \in L^{2}\left(Q_{\alpha}\right)$, define the associated $H_{-1}$ dual norm

$$
\|f\|_{-1}=\inf \left\{\kappa:\langle f, \psi\rangle_{\alpha} \leqslant \kappa\|\psi\|_{1} \text { for all } \psi \in \mathcal{D}\right\}
$$

with usual convention inf $\emptyset=\infty$. We note a large class of functions with finite norm $\|\cdot\|_{-1}$ has been identified in [34] (see also Proposition 3.2). Let $H_{-1}$ be the corresponding Hilbert space completion over $\|\cdot\|_{-1}$ norm finite functions, after modding out by zero-norm quantities.

There are clear relations between $H_{1, \lambda}, H_{-1, \lambda}, H_{1}$ and $H_{-1}$ norms, namely, for $\psi \in \mathcal{D}$ and $f \in L^{2}\left(Q_{\alpha}\right)$,

$$
\|\psi\|_{1} \leqslant\|\psi\|_{1, \lambda} \text { and } \quad\|f\|_{-1, \lambda} \leqslant\|f\|_{-1} .
$$

Consider now the resolvent operator $(\lambda-\mathcal{L})^{-1}: L^{2}\left(Q_{\alpha}\right) \rightarrow \mathbb{D}_{\alpha}$ well defined in terms of the semigroup,

$$
(\lambda-\mathcal{L})^{-1} f=\int_{0}^{\infty} \mathrm{e}^{-\lambda s} T_{s}^{\mathcal{L}} f \mathrm{~d} s .
$$


Define, for $f \in L^{2}\left(Q_{\alpha}\right)$, that $\sigma_{t}^{2}(f)=E_{\alpha}\left[\left(\int_{0}^{t} f(\eta(s)) \mathrm{d} s\right)^{2}\right]$, and observe from the decomposition (1.3), to get diffusive bounds on the tagged particle variance, one need only bound

$$
\sigma_{t}^{2}(\mathfrak{h})=E_{\alpha}\left[\left(\int_{0}^{t} \mathfrak{h}(\eta(s)) \mathrm{d} s\right)^{2}\right]<C t
$$

where $\mathfrak{h}(\eta)=g\left(\eta_{0}\right) / \eta_{0}-\alpha / \rho$ for some constant $C=C(\alpha, g, p, d)$. The next result relates $\sigma_{t}^{2}(f)$ to $H_{-1, \lambda}$ and $H_{-1}$ norms.

Proposition 3.1. For $f \in L^{2}\left(Q_{\alpha}\right)$, and $t>0$, there is a universal constant $C_{1}$ such that

$$
\begin{aligned}
\sigma_{t}^{2}(f) & \leqslant C_{1} t\left\langle f,\left(t^{-1}-\mathcal{L}\right)^{-1} f\right\rangle_{\alpha} \\
& \leqslant C_{1} t\|f\|_{-1, t^{-1}}^{2} \\
& \leqslant C_{1} t\|f\|_{-1}^{2} .
\end{aligned}
$$

Proof. Let $u=(\lambda-\mathcal{L})^{-1} f$. The first line is a computation with the resolvent equation $f=\lambda u-\mathcal{L} u$. First note, by multiplying through by $u$ and integrating, that $\left\langle f,(\lambda-\mathcal{L})^{-1} f\right\rangle_{\alpha}=E_{\alpha}[f u]=\lambda E_{\alpha}\left[u^{2}\right]+E_{\alpha}[u(-\mathcal{L} u)]$. Then, write

$$
\int_{0}^{t} f \mathrm{~d} s=\int_{0}^{t} \lambda u \mathrm{~d} s+M_{\lambda}(t)+u(\eta(0))-u(\eta(t))
$$

where $M_{\lambda}(t)=u(\eta(t))-u(\eta(0))-\int_{0}^{t} \mathcal{L} u \mathrm{~d} s$ is a martingale. Note $\sigma_{t}^{2}(f) \leqslant 3 \sigma_{t}^{2}(\lambda u)+3 E_{\alpha}\left[M_{\lambda}^{2}(t)\right]+3 E_{\alpha}[(u(\eta(0)-$ $\left.u(\eta(t))^{2}\right]$. Using stationarity, $\sigma_{t}^{2}(\lambda u) \leqslant \lambda^{2} t^{2} E_{\alpha}\left[u^{2}\right]$, the quadratic variation $E_{\alpha}\left[M_{\lambda}^{2}(t)\right]=t E_{\alpha}[u(-\mathcal{L} u)]$, and $E_{\alpha}\left[\left(u\left(\eta(0)-u(\eta(t))^{2}\right] \leqslant 4 E_{\alpha}\left[u^{2}\right]\right.\right.$. Hence, by collecting terms, and choosing $\lambda=t^{-1}$, we obtain the first estimate with say $C_{1}=15$ (cf. Section 6, Appendix 1 [10]).

For the second bound, as $u \in \mathbb{D}_{\alpha}$, we can find a sequence of functions $\left\{\psi_{n}\right\} \subset \mathcal{D}$ in the core such that $\psi_{n} \rightarrow u$ and $\mathcal{L} \psi_{n} \rightarrow \mathcal{L} u$ in $L^{2}\left(Q_{\alpha}\right)$. Then, $\left\langle f, \psi_{n}\right\rangle_{\alpha} \rightarrow\left\langle f,(\lambda-\mathcal{L})^{-1} f\right\rangle_{\alpha}$ and $\left\langle\psi_{n},(\lambda-\mathcal{S}) \psi_{n}\right\rangle_{\alpha}=\left\langle\psi_{n},(\lambda-\mathcal{L}) \psi_{n}\right\rangle_{\alpha} \rightarrow$ $\left\langle f,(\lambda-\mathcal{L})^{-1} f\right\rangle_{\alpha}$, and so, by substitution into (3.2), $\left\langle f,(\lambda-\mathcal{L})^{-1} f\right\rangle_{\alpha} \leqslant\|f\|_{-1, \lambda}^{2}$.

The third bound is given in (3.5).

Proof of Theorem 2. The strategy to bound $\sigma_{t}^{2}(\mathfrak{h})$ falls into two cases $d=1$, and $d \geqslant 3$ under (SP). We first comment on the case $d=1$, and then on the $d \geqslant 3$ case.

Case $d=1$. (1) We will find a sequence of functions (in Subsection 3.1.1) $\left\{\phi_{\lambda}: 0<\lambda<1\right\} \subset \mathbb{D}_{\alpha}$ such that

$$
\sup _{0<\lambda<1}\left\|\mathfrak{h}-\mathcal{L} \phi_{\lambda}\right\|_{-1, \lambda}<\infty
$$

and also

$$
\sup _{0<\lambda<1}\left(\left\|\phi_{\lambda}\right\|_{1}^{2}+\lambda\left\|\phi_{\lambda}\right\|_{0}^{2}\right)<\infty .
$$

(2) Note $M_{t}(f)=f(\eta(t))-f(\eta(0))-\int_{0}^{t}(\mathcal{L} f)(\eta(s)) \mathrm{d} s$ is a martingale for $f \in \mathbb{D}_{\alpha}$ with quadratic variation (by stationarity) $E_{\alpha}\left[\left(M_{t}(f)\right)^{2}\right]=2 t E_{\alpha}[f(-\mathcal{L} f)]=2 t\|f\|_{1}^{2}$. Then, we can write

$$
-\int_{0}^{t} \mathcal{L} \phi_{\lambda}(\eta(s)) \mathrm{d} s=M_{t}\left(\phi_{\lambda}\right)+\phi_{\lambda}(\eta(0))-\phi_{\lambda}(\eta(t))
$$

and so (by stationarity)

$$
\sigma_{t}^{2}\left(\mathcal{L} \phi_{\lambda}\right) \leqslant 6\left(t\left\|\phi_{\lambda}\right\|_{1}^{2}+\left\|\phi_{\lambda}\right\|_{0}^{2}\right)=6 t\left(\left\|\phi_{\lambda}\right\|_{1}^{2}+\frac{1}{t}\left\|\phi_{\lambda}\right\|_{0}^{2}\right) .
$$

(3) Hence, by choosing $\lambda=t^{-1}$, we have from Proposition 3.1 that 


$$
\begin{aligned}
\sigma_{t}^{2}(\mathfrak{h}) & \leqslant 2 \sigma_{t}^{2}\left(\mathfrak{h}-\mathcal{L} \phi_{t^{-1}}\right)+2 \sigma_{t}^{2}\left(\mathcal{L} \phi_{t^{-1}}\right) \\
& \leqslant 2 C_{1} t\left\|\mathfrak{h}-\mathcal{L} \phi_{t^{-1}}\right\|_{-1, t^{-1}}^{2}+12 t\left(\left\|\phi_{t^{-1}}\right\|_{1}^{2}+\frac{1}{t}\left\|\phi_{t^{-1}}\right\|_{0}^{2}\right) .
\end{aligned}
$$

Then, by estimates in $(1), \sigma_{t}^{2}(\mathfrak{h}) \leqslant C t$ for some constant $C=C(\alpha, g, p)$ and $t>1$. For $0 \leqslant t \leqslant 1$, bounds are immediate. This finishes the proof in this case.

Case $d \geqslant 3$ and (SP). By Proposition 3.1, we need only show $\|\mathfrak{h}\|_{-1}<\infty$. One may be able to do this directly by "integration-by-parts" but as the $Q_{\alpha}$ marginal at the origin differs from the other marginals, one cannot apply immediately results in the literature. So, we "modify" the function $\mathfrak{h}$ and then apply these results.

Let $j_{0} \neq 0$ be a point in the support of $p$ where $p\left(j_{0}\right) \neq 0$. Consider the function $\phi(\eta)=\left(\eta_{j_{0}}-\rho\right) /\left(\rho p\left(j_{0}\right)\right) \in \mathcal{D}$. In Subsection 3.1.2, we show that $\|\mathfrak{h}-\mathcal{L} \phi\|_{-1}<\infty$. Clearly $\|\phi\|_{1}<\infty$ and $\|\phi\|_{0}<\infty$. Then, by following the sequence (3.8) (noting $\|\cdot\|_{-1, \lambda} \leqslant\|\cdot\|_{-1}$ ), we have

$$
\sigma_{t}^{2}(\mathfrak{h}) \leqslant 2 C_{1} t\|\mathfrak{h}-\mathcal{L} \phi\|_{-1}+12 t\left(\|\phi\|_{1}^{2}+\frac{1}{t}\|\phi\|_{0}^{2}\right)<C t
$$

for a constant $C=C(\alpha, g, p, d)$ and $t>1$. Bounds when $0 \leqslant t \leqslant 1$ are clear. This finishes the proof.

\subsection{Some estimates}

We now turn to supplying the needed estimates in the two cases. We first make a calculation valid in any dimension $d \geqslant 1$. Let $\left\{a_{i}: i \in \mathbb{Z}^{d}\right\}$ be numbers with $\sum\left|a_{i}\right|<\infty$, and define

$$
\phi(\eta)=\sum_{i \in \mathbb{Z}^{d}} a_{i}\left(\eta_{i}-\rho\right) .
$$

Note that $\phi$ absolutely converges $Q_{\alpha}$ a.s. and, as $\sum a_{i}^{2}<\infty$, that also $\phi$ is the $L^{2}\left(Q_{\alpha}\right) \operatorname{limit} \phi=\lim _{n \rightarrow \infty} \phi^{(n)}$ of functions $\phi^{(n)}=\sum_{|i|_{\max } \leqslant n} a_{i}\left(\eta_{i}-\rho\right) \in \mathcal{D}$ where $|i|_{\max }=\max \left\{\left|i_{1}\right|, \ldots,\left|i_{d}\right|\right\}$. Consider now, $n>R$ larger than the range of $p$, and $i, j$ where $|i|_{\max },|i+j|_{\max } \leqslant n$, that

$$
\phi^{(n)}\left(\eta^{i, i+j}\right)-\phi^{(n)}(\eta)=a_{i+j}-a_{i}
$$

and, as $\tau_{j}\left(\eta^{0, j}\right)=\tau_{j}\left(\eta+\delta_{j}-\delta_{0}\right)=\tau_{j} \eta+\delta_{0}-\delta_{-j}$, that $\phi^{(n)}\left(\tau_{j}\left(\eta^{0, j}\right)\right)=\sum_{|i|_{\max } \leqslant n} a_{i}\left(\eta_{i+j}-\rho\right)+a_{0}-a_{-j}$ and

$$
\begin{aligned}
\phi^{(n)}\left(\tau_{j}\left(\eta^{0, j}\right)\right)-\phi^{(n)}(\eta)= & \sum_{\substack{|i|_{\max } \leqslant n \\
|i+j|_{\max } \leqslant n}}\left(a_{i}-a_{i+j}\right)\left(\eta_{i+j}-\rho\right)+\left(a_{0}-a_{-j}\right) \\
& +\sum_{\substack{\left.|i|\right|_{\max } \leqslant n \\
|i+j| \max >n}} a_{i}\left(\eta_{i+j}-\rho\right)-\sum_{\substack{|i| \max >n \\
|i+j|_{\max } \leqslant n}} a_{i+j}\left(\eta_{i+j}-\rho\right) .
\end{aligned}
$$

These computations allow us to write

$$
\begin{aligned}
\left(\mathcal{L} \phi^{(n)}\right)(\eta)= & \sum_{j} \sum_{\substack{i \neq 0,|i|_{\max } \leqslant\left. n \\
|i+j|\right|_{\max } \leqslant n}}\left(a_{i+j}-a_{i}\right) g\left(\eta_{i}\right) p(j)+\sum_{j}\left(a_{j}-a_{0}\right) g\left(\eta_{0}\right) \frac{\eta_{0}-1}{\eta_{0}} p(j) \\
& -\sum_{j} \sum_{\substack{|i|_{\max } \leqslant n \\
|i+j|_{\max } \leqslant n}}\left(a_{i+j}-a_{i}\right)\left(\eta_{i+j}-\rho\right) \frac{g\left(\eta_{0}\right)}{\eta_{0}} p(j)+\sum_{j}\left(a_{0}-a_{-j}\right) \frac{g\left(\eta_{0}\right)}{\eta_{0}} p(j) \\
& -\sum_{j} \sum_{\substack{|i|_{\max } \leqslant n \\
|i+j|_{\max }>n}} a_{i}\left(g\left(\eta_{i}\right)-\left(\eta_{i+j}-\rho\right) \frac{g\left(\eta_{0}\right)}{\eta_{0}}\right) p(j) \\
& +\sum_{j} \sum_{\substack{|i|_{\max }>n \\
|i+j|_{\max } \leqslant n}} a_{i+j}\left(g\left(\eta_{i}\right)-\left(\eta_{i+j}-\rho\right) \frac{g\left(\eta_{0}\right)}{\eta_{0}}\right) p(j)
\end{aligned}
$$


where the last two lines correspond to boundary terms. As $E_{\alpha}\left[\mathcal{L} \phi^{(n)}\right]=0$, we can subtract the mean from all terms, and deduce, from orthogonality relations and the finite-range of $p$, that the $L^{2}\left(Q_{\alpha}\right) \operatorname{limit}_{\lim } \rightarrow \infty \mathcal{L} \phi^{(n)}=\Phi$ converges, and so, as $\mathcal{L}$ is closed, that $\phi \in \mathbb{D}_{\alpha}$ and $\Phi=\mathcal{L} \phi$. Moreover, we have

$$
\begin{aligned}
(\mathcal{L} \phi)(\eta)= & \sum_{j} \sum_{i \neq 0}\left(a_{i+j}-a_{i}\right) g\left(\eta_{i}\right) p(j)+\sum_{j}\left(a_{j}-a_{0}\right) g\left(\eta_{0}\right) \frac{\eta_{0}-1}{\eta_{0}} p(j) \\
& -\sum_{j} \sum_{i}\left(a_{i+j}-a_{i}\right)\left(\eta_{i+j}-\rho\right) \frac{g\left(\eta_{0}\right)}{\eta_{0}} p(j)+\sum_{j}\left(a_{0}-a_{-j}\right) \frac{g\left(\eta_{0}\right)}{\eta_{0}} p(j) \\
= & \sum_{j} \sum_{i \neq 0,-j}\left(a_{i+j}-a_{i}\right)\left[g\left(\eta_{i}\right)-\eta_{i+j} \frac{g\left(\eta_{0}\right)}{\eta_{0}}\right] p(j)+\sum_{j}\left(a_{0}-a_{-j}\right)\left[g\left(\eta_{-j}\right)-g\left(\eta_{0}\right) \frac{\eta_{0}-1}{\eta_{0}}\right] p(j) \\
& +\sum_{j}\left(a_{j}-a_{0}\right) g\left(\eta_{0}\right)\left[1-\frac{\eta_{j}+1}{\eta_{0}}\right] p(j) .
\end{aligned}
$$

Here, we used $\sum_{j} \sum_{i}\left(a_{i+j}-a_{i}\right) p(j)=0$ to reduce the first sum in the second line to

$$
-\sum_{j} \sum_{i}\left(a_{i+j}-a_{i}\right) \eta_{i+j}\left(\frac{g\left(\eta_{0}\right)}{\eta_{0}}\right) p(j)
$$

We remark in the following, we could work with $\phi^{(n)}$ itself but going to the limit here avoids treating boundary terms later on.

We now note the following basic useful relations.

Lemma 3.1. Let $j \in \mathbb{Z}^{d}$ be a non-zero vertex, $j \neq 0$. Then, for $\psi \in L^{2}\left(Q_{\alpha}\right)$, we have

$$
E_{\alpha}\left[g\left(\eta_{j}\right) \psi(\eta)\right]=\alpha E_{\alpha}\left[\psi\left(\eta+\delta_{j}\right)\right], \quad E_{\alpha}\left[g\left(\eta_{0}\right) \frac{\eta_{0}-1}{\eta_{0}} \psi(\eta)\right]=\alpha E_{\alpha}\left[\psi\left(\eta+\delta_{0}\right)\right],
$$

and

$$
E_{\alpha}\left[\frac{g\left(\eta_{0}\right)}{\eta_{0}} \psi(\eta)\right]=E_{\alpha}\left[\frac{g\left(\eta_{0}\right)}{\eta_{0}} \psi\left(\tau_{j}\left(\eta^{0, j}\right)\right)\right], \quad E_{\alpha}\left[\left(\eta_{j}+1\right) \frac{g\left(\eta_{0}\right)}{\eta_{0}} \psi(\eta)\right]=E_{\alpha}\left[g\left(\eta_{0}\right) \psi\left(\tau_{-j}\left(\eta^{0,-j}\right)\right)\right] .
$$

Proof. We show the last equality as the others are similar. Write

$$
\begin{aligned}
E_{\alpha}\left[\left(\eta_{j}+1\right) \frac{g\left(\eta_{0}\right)}{\eta_{0}} \psi(\eta)\right] & =\frac{\alpha}{\rho} E_{R_{\alpha}}\left[\left(\eta_{j}+1\right) \psi\left(\eta+\delta_{0}\right)\right]=\frac{1}{\rho} E_{R_{\alpha}}\left[g\left(\eta_{j}\right) \eta_{j} \psi\left(\eta^{j, 0}\right)\right] \\
& =E_{\alpha}\left[g\left(\eta_{0}\right) \psi\left(\left(\tau_{-j} \eta\right)^{j, 0}\right)\right]=E_{\alpha}\left[g\left(\eta_{0}\right) \psi\left(\tau_{-j}\left(\eta^{0,-j}\right)\right)\right] .
\end{aligned}
$$

Let now $\psi \in \mathcal{D}$ be a function. We can write, with Lemma 3.1,

$$
\begin{aligned}
E_{\alpha}[(\mathcal{L} \phi) \psi]= & \sum_{j} \sum_{i \neq 0,-j}\left(a_{i+j}-a_{i}\right) E_{\alpha}\left[\left(g\left(\eta_{i}\right)-\eta_{i+j} \frac{g\left(\eta_{0}\right)}{\eta_{0}}\right) \psi(\eta)\right] p(j) \\
& +\alpha \sum_{j}\left(a_{0}-a_{-j}\right) E_{\alpha}\left[\psi\left(\eta+\delta_{-j}\right)-\psi\left(\eta+\delta_{0}\right)\right] p(j) \\
& +\sum_{j}\left(a_{j}-a_{0}\right) E_{\alpha}\left[g\left(\eta_{0}\right)\left(\psi(\eta)-\psi\left(\tau_{-j}\left(\eta^{0,-j}\right)\right)\right)\right] p(j) .
\end{aligned}
$$

It will be convenient, for later purposes, to observe that in the above computation we can take $E_{\alpha}[\psi]=0$ without loss of generality as $E_{\alpha}[\mathcal{L} \phi]=0$. 


\subsubsection{Estimates in $d=1$}

We now work in dimension $d=1$, and choose the sequence

$$
a_{i}= \begin{cases}0 & \text { for } i \leqslant 0 \\ c_{0}(1-\lambda)^{i-1} & \text { for } i \geqslant 1\end{cases}
$$

with $c_{0}=\left(\rho \sum j p(j)\right)^{-1}$ and $0<\lambda<1$. Then, $\phi$ now also depends on $\lambda, \phi=\phi_{\lambda}$. For ease of notation, define $\nabla a_{k, j}=a_{k}-a_{j}$ and note

$$
\nabla a_{i+j, i}= \begin{cases}0 & \text { for } i, i+j \leqslant 0, \\ c_{0}(1-\lambda)^{i+j-1} & \text { for } i+j \geqslant 1 \text { and } i \leqslant 0, \\ -c_{0}(1-\lambda)^{i-1} & \text { for } i \geqslant 1 \text { and } i+j \leqslant 0, \\ c_{0}\left[(1-\lambda)^{j}-1\right](1-\lambda)^{i-1} & \text { for } i, i+j \geqslant 1 .\end{cases}
$$

Clearly $\left|\nabla a_{i+j, i}\right| \leqslant\left|c_{0}\right|$ for all $i, j \in \mathbb{Z}$.

Recall now the range $R$ of the distribution $p$, and write, following (3.9),

$$
\begin{aligned}
E_{\alpha}[(\mathcal{L} \phi) \psi]= & \sum_{j} \sum_{i \geqslant R+2} \nabla a_{i+j, i} E_{\alpha}\left[\left(g\left(\eta_{i}\right)-\eta_{i+j} \frac{g\left(\eta_{0}\right)}{\eta_{0}}\right) \psi(\eta)\right] p(j) \\
& +\sum_{j} \sum_{\substack{|i| \leqslant R+1 \\
i \neq 0,-j}} \nabla a_{i+j, i} E_{\alpha}\left[\left(g\left(\eta_{i}\right)-\eta_{i+j} \frac{g\left(\eta_{0}\right)}{\eta_{0}}\right) \psi(\eta)\right] p(j) \\
& +\sum_{j} \nabla a_{0,-j} E_{\alpha}\left[g\left(\eta_{0}\right) \frac{\eta_{0}-1}{\eta_{0}}\left(\psi\left(\eta^{0,-j}\right)-\psi(\eta)\right)\right] p(j) \\
& +\sum_{j} \nabla a_{j, 0} E_{\alpha}\left[g\left(\eta_{0}\right)\left(\psi(\eta)-\psi\left(\tau_{-j}\left(\eta^{0,-j}\right)\right)\right)\right] p(j) \\
= & I_{1}+I_{2}+I_{3}+I_{4} .
\end{aligned}
$$

Consider now the term $I_{1}$. Since $E_{\alpha}[\psi]=0$, we can write

$$
\begin{aligned}
I_{1}= & \sum_{j} \sum_{i \geqslant R+2} \nabla a_{i+j, i} E_{\alpha}\left[\left(\left(g\left(\eta_{i}\right)-\alpha\right)-\left(\eta_{i+j}-\rho\right) \frac{g\left(\eta_{0}\right)}{\eta_{0}}\right) \psi(\eta)\right] p(j) \\
& -\rho \sum_{j} \sum_{i \geqslant R+2} \nabla a_{i+j, i} E_{\alpha}\left[\frac{g\left(\eta_{0}\right)}{\eta_{0}} \psi(\eta)\right] p(j) \\
= & J_{1}+J_{2} .
\end{aligned}
$$

After a calculation, the last term $J_{2}$ equals, using $E_{\alpha}[\psi]=0$ again and that $p(0)=0$,

$$
\begin{aligned}
J_{2}= & \rho E_{\alpha}\left[\frac{g\left(\eta_{0}\right)}{\eta_{0}} \psi(\eta)\right]\left\{\sum_{j \geqslant 1} \sum_{k=2}^{1+j} a_{R+k} p(j)-\sum_{j \leqslant-1} \sum_{k=2+j}^{1} a_{R+k} p(j)\right\} \\
= & \rho c_{0} E_{\alpha}\left[\frac{g\left(\eta_{0}\right)}{\eta_{0}} \psi(\eta)\right]\left\{\sum_{j \geqslant 1} p(j)(1-\lambda)^{R+1} \sum_{k=0}^{j-1}(1-\lambda)^{k}-\sum_{j \leqslant-1} p(j)(1-\lambda)^{R+1+j} \sum_{k=0}^{-1-j}(1-\lambda)^{k}\right\} \\
= & \rho c_{0}\left(\sum j p(j)\right) E_{\alpha}\left[\frac{g\left(\eta_{0}\right)}{\eta_{0}} \psi(\eta)\right]+\rho c_{0} E_{\alpha}\left[\frac{g\left(\eta_{0}\right)}{\eta_{0}} \psi(\eta)\right]\left\{\sum_{j \geqslant 1} p(j)\left[(1-\lambda)^{R+1} \frac{1-(1-\lambda)^{j}}{\lambda}-j\right]\right. \\
& \left.-\sum_{j \leqslant-1} p(j)\left[(1-\lambda)^{R+1+j} \frac{1-(1-\lambda)^{-j}}{\lambda}+j\right]\right\} \\
= & E_{\alpha}[\mathfrak{h} \psi(\eta)]+J_{3} .
\end{aligned}
$$


Hence, we have that

$$
E_{\alpha}[(\mathfrak{h}-\mathcal{L} \phi) \psi]=-\left(I_{2}+I_{3}+I_{4}+J_{1}+J_{3}\right) .
$$

To show the bound in (3.6), by the variational form of $\|\cdot\|_{-1, \lambda}$ (cf. (3.2)), we need only verify

$$
\left|I_{2}+I_{3}+I_{4}+J_{1}+J_{3}\right| \leqslant C\|\psi\|_{1, \lambda}
$$

for some constant $C=C(\alpha, g, p)$.

To this end, observe, by Schwarz inequality, using $E_{\alpha}\left[g\left(\eta_{0}\right)\left(\eta_{0}-1\right) / \eta_{0}\right]=\alpha$ and $p(j) \leqslant 2 s(-j)$, that

$$
\left|I_{3}\right| \leqslant\left(\sum_{j}\left|c_{0}\right|^{2} p(j)\right)^{1 / 2}\left(\sum_{j} \alpha E_{\alpha}\left[g\left(\eta_{0}\right) \frac{\eta_{0}-1}{\eta_{0}}\left(\psi\left(\eta^{0,-j}\right)-\psi(\eta)\right)^{2}\right] p(j)\right)^{1 / 2} \leqslant C\|\psi\|_{1}
$$

for a constant $C=C(\alpha, g, p)$ noting (3.1). Similarly,

$$
\begin{aligned}
\left|I_{4}\right| & \leqslant\left(\sum_{j}\left|c_{0}\right|^{2} p(j)\right)^{1 / 2}\left(\sum_{j} E_{\alpha}\left[g\left(\eta_{0}\right) \eta_{0}\right] E_{\alpha}\left[\frac{g\left(\eta_{0}\right)}{\eta_{0}}\left(\psi(\eta)-\psi\left(\tau_{-j}\left(\eta^{0,-j}\right)\right)\right)^{2}\right] p(j)\right)^{1 / 2} \\
& \leqslant 2\left|c_{0}\right| \sqrt{E_{\alpha}\left[g\left(\eta_{0}\right) \eta_{0}\right]}\|\psi\|_{1} .
\end{aligned}
$$

For the second term $I_{2}$, note, with Lemma 3.1,

$$
\begin{aligned}
E_{\alpha} & {\left[\left(g\left(\eta_{i}\right)-\eta_{i+j} \frac{g\left(\eta_{0}\right)}{\eta_{0}}\right) \psi(\eta)\right] } \\
& =E_{\alpha}\left[g\left(\eta_{0}\right) \frac{\eta_{0}-1}{\eta_{0}}\left(\psi\left(\eta^{0, i}\right)-\psi(\eta)\right)+\left(g\left(\eta_{0}\right)-g\left(\eta_{0}\right) \frac{\eta_{i+j}+1}{\eta_{0}}\right) \psi(\eta)\right] \\
& =E_{\alpha}\left[g\left(\eta_{0}\right) \frac{\eta_{0}-1}{\eta_{0}}\left(\psi\left(\eta^{0, i}\right)-\psi(\eta)\right)+g\left(\eta_{0}\right)\left(\psi(\eta)-\psi\left(\tau_{-(i+j)}\left(\eta^{0,-(i+j)}\right)\right)\right)\right] .
\end{aligned}
$$

Then,

$$
\begin{aligned}
\left|I_{2}\right| \leqslant & \left(\sum_{j} \sum_{\substack{|i| \leqslant R+1 \\
i \neq 0,-j}}\left|c_{0}\right|^{2} p(j)\right)^{1 / 2}\left(\sum_{j} \sum_{|i| \leqslant R+1} 2 \alpha E_{\alpha}\left[g\left(\eta_{0}\right) \frac{\eta_{0}-1}{\eta_{0}}\left(\psi\left(\eta^{0, i}\right)-\psi(\eta)\right)^{2}\right] p(j)\right. \\
& \left.+2 E_{\alpha}\left[g\left(\eta_{0}\right) \eta_{0}\right] E_{\alpha}\left[\frac{g\left(\eta_{0}\right)}{\eta_{0}}\left(\psi(\eta)-\psi\left(\tau_{-(i+j)}\left(\eta^{0,-(i+j)}\right)\right)\right)^{2}\right] p(j)\right)^{1 / 2} \cdot
\end{aligned}
$$

Note, as $s$ is irreducible, $u \in \mathbb{Z}$ can be written $u=\sum_{n=1}^{m} l_{n}$ for points $l_{n}$ in the support of $s, s\left(l_{n}\right)>0$. Let $r_{0}=0$ and $r_{k}=\sum_{n=1}^{k} l_{n}$ for $1 \leqslant k \leqslant m$. Then, with Lemma 3.1,

$$
\begin{aligned}
E_{\alpha}\left[g\left(\eta_{0}\right) \frac{\eta_{0}-1}{\eta_{0}}\left(\psi\left(\eta^{0, u}\right)-\psi(\eta)\right)^{2}\right] & =\alpha E_{\alpha}\left[\left(\psi\left(\eta+\delta_{u}\right)-\psi\left(\eta+\delta_{0}\right)\right)^{2}\right] \\
& \leqslant m \alpha \sum_{k=0}^{m-1} E_{\alpha}\left[\left(\psi\left(\eta+\delta_{r_{k}}\right)-\psi\left(\eta+\delta_{r_{k+1}}\right)\right)^{2}\right] \\
& \leqslant C\|\psi\|_{1}^{2}
\end{aligned}
$$

for some constant $C=C(p)$ as $\min _{i: p(i)>0} p(i)>0$ given $p$ is finite-range. Also,

$$
\begin{aligned}
E_{\alpha}\left[\frac{g\left(\eta_{0}\right)}{\eta_{0}}\left(\psi(\eta)-\psi\left(\tau_{u}\left(\eta^{0, u}\right)\right)\right)^{2}\right] & \leqslant m \sum_{k=0}^{m-1} E_{\alpha}\left[\frac{g\left(\eta_{0}\right)}{\eta_{0}}\left(\psi\left(\tau_{r_{k}}\left(\eta^{0, r_{k}}\right)\right)-\psi\left(\tau_{r_{k+1}}\left(\eta^{0, r_{k+1}}\right)\right)\right)^{2}\right] \\
& =m \sum_{k=0}^{m-1} E_{\alpha}\left[\frac{g\left(\eta_{0}\right)}{\eta_{0}}\left(\psi(\eta)-\psi\left(\tau_{l_{k+1}}\left(\eta^{0, l_{k+1}}\right)\right)\right)^{2}\right] \leqslant C\|\psi\|_{1}^{2}
\end{aligned}
$$


for a $C=C(p)$ again as $p$ is finite-range. Then, as the sums in $I_{2}$ are finite, $I_{2}$ is bounded $\left|I_{2}\right| \leqslant C\|\psi\|_{1}$ for some constant $C=C(\alpha, g, p)$.

To bound $J_{1}$, we use the resolvent bound (3.3). Namely, as $\left\{g\left(\eta_{i}\right)-\alpha: i \geqslant R+2\right\}$ is an orthogonal family,

$$
\begin{aligned}
\left|\sum_{j} \sum_{i \geqslant R+2} \nabla a_{i+j, i} E_{\alpha}\left[\left(g\left(\eta_{i}\right)-\alpha\right) \psi(\eta)\right] p(j)\right| & \leqslant\left\|\sum_{j} \sum_{i \geqslant R+2} \nabla a_{i+j, i}\left(g\left(\eta_{i}\right)-\alpha\right) p(j)\right\|_{-1, \lambda}\|\psi\|_{1, \lambda} \\
& \leqslant\left(\frac{1}{\lambda} \sum_{|j| \leqslant R} \sum_{i \geqslant R+2} \nabla^{2} a_{i+j, i} E_{\alpha}\left[\left(g\left(\eta_{1}\right)-\alpha\right)^{2}\right] p(j)\right)^{1 / 2}\|\psi\|_{1, \lambda}
\end{aligned}
$$

and, as for fixed $j,\left\{\left(\eta_{i+j}-\rho\right)\left(g\left(\eta_{0}\right) / \eta_{0}\right): i \geqslant R+2\right\}$ is also an orthogonal collection,

$$
\begin{aligned}
& \left|\sum_{j} \sum_{i \geqslant R+2} \nabla a_{i+j, i} E_{\alpha}\left[\left(\eta_{i+j}-\rho\right) \frac{g\left(\eta_{0}\right)}{\eta_{0}} \psi(\eta)\right] p(j)\right| \\
& \quad \leqslant\left(\frac{1}{\lambda} \sum_{|j| \leqslant R} \sum_{i \geqslant R+2} \nabla^{2} a_{i+j, i} E_{\alpha}\left[\left(\eta_{1}-\rho\right)^{2} \frac{g^{2}\left(\eta_{0}\right)}{\eta_{0}^{2}}\right] p(j)\right)^{1 / 2}\|\psi\|_{1, \lambda} ;
\end{aligned}
$$

then, $\left|J_{1}\right| \leqslant C\left(\lambda^{-1} \sum_{i \geqslant 1} \nabla^{2} a_{i+1, i}\right)^{1 / 2}\|\psi\|_{1, \lambda}$ for some $C=C(\alpha, g, p)$ as $i+j \geqslant 2$ for $i \geqslant R+2$ and $|j| \leqslant R$.

Finally, $J_{3}$ is uniformly bounded through the resolvent bound (3.3) for $0<\lambda<1$ with respect to a constant $C=$ $C(\alpha, g, p)$ as

$$
\begin{aligned}
\left|J_{3}\right| \leqslant & {\left[\frac { ( \rho c _ { 0 } ) ^ { 2 } } { \lambda } \left\{\sum_{1 \leqslant j \leqslant R} p(j)\left[(1-\lambda)^{R+1} \frac{1-(1-\lambda)^{j}}{\lambda}-j\right]\right.\right.} \\
& \left.\left.-\sum_{-R \leqslant j \leqslant-1} p(j)\left[(1-\lambda)^{R+1+j} \frac{1-(1-\lambda)^{-j}}{\lambda}+j\right]\right\}^{2}\left\|\frac{g\left(\eta_{0}\right)}{\eta_{0}}\right\|_{0}^{2}\right]^{1 / 2}\|\psi\|_{1, \lambda} \\
\leqslant & C\|\psi\|_{1, \lambda .}
\end{aligned}
$$

Putting these estimates together, noting $\|\psi\|_{1} \leqslant\|\psi\|_{1, \lambda}$ and using a form of Schwarz - relation $2 a b=\inf _{\epsilon} \epsilon^{-1} a^{2}+$ $\epsilon b^{2}-$ we obtain, for a constant $C=C(\alpha, g, p)$,

$$
\left|I_{2}+I_{3}+I_{4}+J_{1}+J_{3}\right| \leqslant C\left(1+\frac{1}{\lambda} \sum_{i \geqslant 1} \nabla^{2} a_{i+1, i}\right)^{1 / 2}\|\psi\|_{1, \lambda} .
$$

Then, by direct computation, we have that

$$
\frac{1}{\lambda} \sum_{i \geqslant 1} \nabla^{2} a_{i+1, i}=\frac{\lambda^{2}\left|c_{0}\right|^{2}}{\lambda} \frac{1}{\lambda(2-\lambda)}
$$

which shows (3.6) via (3.10).

To show (3.7), we observe $\sum_{i \geqslant 1} a_{i}^{2}=\left|c_{0}\right|^{2} /(\lambda(2-\lambda))$ and so $\lambda\|\phi\|_{0}^{2}=\lambda\left(\left|c_{0}\right|^{2} /(\lambda(2-\lambda))\right) E_{\alpha}\left[\left(\eta_{1}-\rho\right)^{2}\right]$; also, noting computations at the beginning of Subsection 3.1 and (3.1), we have uniformly over $0<\lambda<1$ that

$$
\begin{aligned}
\|\phi\|_{1}^{2} & =\frac{\alpha}{2} \sum_{j} \sum_{i} \nabla^{2} a_{i+j, i} s(j)+\frac{1}{2} \sum_{j} E_{\alpha}\left[\frac{g\left(\eta_{0}\right)}{\eta_{0}}\left(\sum_{i} \nabla a_{i, i+j}\left(\eta_{i+j}-\rho\right)+\nabla a_{0,-j}\right)^{2}\right] s(j) \\
& \leqslant C+C \sum_{i \geqslant 1} \nabla^{2} a_{i+1, i} \leqslant C^{\prime}
\end{aligned}
$$

for some constants $C=C(\alpha, g, p)$ and $C^{\prime}=C^{\prime}(\alpha, g, p)$ using, as before, the orthogonality of

$$
\left\{\left(\frac{g\left(\eta_{0}\right)}{\eta_{0}}\right)\left(\eta_{i+j}-\rho\right): i \in \mathbb{Z}\right\}
$$

for fixed $j$, and that $s(\cdot)$ is finite-range. 


\subsubsection{Estimates in $d \geqslant 3$ under $(S P)$}

For the function $\phi(\eta)=\left(\eta_{j_{0}}-\rho\right) /\left(\rho p\left(j_{0}\right)\right)$ and $\psi \in \mathcal{D}$, we have from (3.9), noting here $a_{j_{0}}=1$ and $a_{i}=0$ for $i \neq j_{0}$, and also $j_{0} \neq 0$, that $\rho p\left(j_{0}\right) E_{\alpha}[(\mathcal{L} \phi) \psi]$ equals

$$
\begin{aligned}
& -E_{\alpha}\left[\left(g\left(\eta_{j_{0}}\right)-\eta_{2 j_{0}} \frac{g\left(\eta_{0}\right)}{\eta_{0}}\right) \psi\right] p\left(j_{0}\right)+E_{\alpha}\left[\left(g\left(\eta_{2 j_{0}}\right)-\eta_{j_{0}} \frac{g\left(\eta_{0}\right)}{\eta_{0}}\right) \psi\right] p\left(-j_{0}\right) \\
& \quad+\sum_{j \neq \pm j_{0}}\left\{E_{\alpha}\left[\left(g\left(\eta_{j_{0}-j}\right)-\eta_{j_{0}} \frac{g\left(\eta_{0}\right)}{\eta_{0}}\right) \psi\right]-E_{\alpha}\left[\left(g\left(\eta_{j_{0}}\right)-\eta_{j_{0}+j} \frac{g\left(\eta_{0}\right)}{\eta_{0}}\right) \psi\right]\right\} p(j) \\
& -\alpha E_{\alpha}\left[\psi\left(\eta+\delta_{j_{0}}\right)-\psi\left(\eta+\delta_{0}\right)\right] p\left(-j_{0}\right)+E_{\alpha}\left[g\left(\eta_{0}\right)\left(\psi(\eta)-\psi\left(\tau_{-j_{0}}\left(\eta^{0,-j_{0}}\right)\right)\right)\right] p\left(j_{0}\right) .
\end{aligned}
$$

As we can take $E_{\alpha}[\psi]=0$, without loss of generality, with Lemma 3.1, $\rho p\left(j_{0}\right) E_{\alpha}[(\mathcal{L} \phi) \psi]$ equals

$$
\begin{aligned}
& \rho p\left(j_{0}\right) E_{\alpha}\left[\frac{g\left(\eta_{0}\right)}{\eta_{0}} \psi\right]-E_{\alpha}\left[\left(g\left(\eta_{j_{0}}\right)-\alpha\right) \psi\right] p\left(j_{0}\right)+E_{\alpha}\left[\left(\eta_{2 j_{0}}-\rho\right) \frac{g\left(\eta_{0}\right)}{\eta_{0}} \psi\right] p\left(j_{0}\right) \\
& \quad+E_{\alpha}\left[\left(g\left(\eta_{2 j_{0}}\right)-\eta_{j_{0}} \frac{g\left(\eta_{0}\right)}{\eta_{0}}\right) \psi\right] p\left(-j_{0}\right)+\sum_{\substack{j \neq \pm j_{0} \\
|j| \leqslant R}}\left\{E_{\alpha}\left[\left(g\left(\eta_{j_{0}-j}\right)-\eta_{j_{0}} \frac{g\left(\eta_{0}\right)}{\eta_{0}}\right) \psi\right]\right. \\
& \left.\quad-E_{\alpha}\left[\left(g\left(\eta_{j_{0}}\right)-\eta_{j_{0}+j} \frac{g\left(\eta_{0}\right)}{\eta_{0}}\right) \psi\right]\right\} p(j)-E_{\alpha}\left[g\left(\eta_{0}\right) \frac{\eta_{0}-1}{\eta_{0}}\left(\psi\left(\eta^{0, j_{0}}\right)-\psi(\eta)\right)\right] p\left(-j_{0}\right) \\
& \quad+E_{\alpha}\left[g\left(\eta_{0}\right)\left(\psi(\eta)-\psi\left(\tau_{-j_{0}}\left(\eta^{0,-j_{0}}\right)\right)\right)\right] p\left(j_{0}\right) \\
& =\rho p\left(j_{0}\right) E_{\alpha}\left[\frac{g\left(\eta_{0}\right)}{\eta_{0}} \psi\right]+K_{1}+K_{2}+K_{3}+K_{4}+K_{5}+K_{6} .
\end{aligned}
$$

Hence, to show $\|\mathfrak{h}-\mathcal{L} \phi\|_{-1}<\infty$, by the variational characterization (cf. (3.4)), we need only show that

$$
\left|E_{\alpha}[(\mathfrak{h}-\mathcal{L} \phi) \psi]\right|=\left(\rho p\left(j_{0}\right)\right)^{-1}\left|K_{1}+K_{2}+K_{3}+K_{4}+K_{5}+K_{6}\right| \leqslant C\|\psi\|_{1}
$$

for some constant $C=C(\alpha, g, p)$. To this end, the terms $K_{3}, K_{4}, K_{5}$ and $K_{6}$ are handled analogously as $I_{2}, I_{3}$ and $I_{4}$ above in the $d=1$ case. To bound $K_{1}$ and $K_{2}$, we invoke the following result.

Proposition 3.2. Consider $d \geqslant 3$ finite-range reference frame processes such that $g$ satisfies assumption (SP). Let $f$ be a $L^{4}\left(Q_{\alpha}\right)$ function supported on a finite number of vertices of $\mathbb{Z}^{d} \backslash\{0\}$ which is mean-zero, $E_{\alpha}[f]=0$. Then, for a constant $C=C(f, \alpha, g, p, d)$, we have for $\psi \in \mathcal{D}$ that

$$
E_{\alpha}[f \psi] \leqslant C\left[\sum_{j} \sum_{i \neq 0,-j} E_{\alpha}\left[g\left(\eta_{i}\right)\left(\psi\left(\eta^{i, i+j}\right)-\psi(\eta)\right)^{2}\right] s(j)\right]^{1 / 2} \leqslant C\|\psi\|_{1} .
$$

In particular, $\|f\|_{-1}<\infty$.

Proof. The proof is virtually the same as for Theorem 1.2 [34] and accomplished by straightforwardly avoiding the origin.

Note now Proposition 3.2 directly applies to $K_{1}$. For $K_{2}$, we first condition on $\eta_{0}$, noting $Q_{\alpha}$ is product measure, to get

$$
\begin{aligned}
\left|K_{2}\right| & =p\left(j_{0}\right)\left|E_{\alpha}\left[E_{\alpha}\left[\left(\eta_{2 j_{0}}-\rho\right) \psi\left(\cdot ; \eta_{0}\right) \mid \eta_{0}\right] \frac{g\left(\eta_{0}\right)}{\eta_{0}}\right]\right| \\
& \leqslant p\left(j_{0}\right) E_{\alpha}\left[C\left[\sum_{j} \sum_{i \neq 0,-j} E_{\alpha}\left[g\left(\eta_{i}\right)\left(\psi\left(\eta^{i, i+j} ; \eta_{0}\right)-\psi\left(\eta ; \eta_{0}\right)\right)^{2}\right] s(j)\right]^{1 / 2} \frac{g\left(\eta_{0}\right)}{\eta_{0}}\right]
\end{aligned}
$$

where $\psi\left(\chi ; \eta_{0}\right)$ denotes $\psi$ as a function of $\left\{\chi_{i}: i \neq 0\right\}$ with $\eta_{0}$ fixed. After applying Schwarz inequality, as $Q_{\alpha}$ is product measure, one can integrate and bound the right-side by $C p\left(j_{0}\right) E_{\alpha}\left[\left(g\left(\eta_{0}\right) / \eta_{0}\right)^{2}\right]^{1 / 2}\|\psi\|_{1}$. This finishes the proof of Theorem 2 in this case. 


\section{Proof of Theorem 3}

We first define a notion of a "weakly positively associated" stationary increments $L^{2}$ process $N(t)$. This is an $L^{2}$ process where

$$
E[\phi(N(t+s)-N(t)) \psi(N(t))] \geqslant E[\phi(N(s))] E[\psi(N(t))]
$$

for all $\phi$ and $\psi$ increasing. For such processes we have the Newman-Wright result (cf. [21] for a survey).

Theorem 4. Suppose $N(t)$ is an $L^{2}$ process on $D[0, \infty)$ with weakly positively associated stationary increments such that the limit variance is finite

$$
\lim _{t \rightarrow \infty} \frac{1}{t} E\left[(N(t)-E[N(t)])^{2}\right]=\sigma^{2}<\infty
$$

Then, we have finite-dimensional convergence to a Brownian motion $\mathbb{B}$ with diffusion coefficient $\sigma^{2}$,

$$
\frac{1}{\sqrt{\lambda}}(N(\lambda t)-E[N(\lambda t)]) \Rightarrow \mathbb{B}(t)
$$

Proof. The result is Theorem 3 [20] applied in our context. We note that although Theorem 3 [20] assumes a stronger form of associativity, what is actually used in the proof is the notion of "weakly positive associativity" defined above.

Remark. We note from the assumption of stationary and positive association of increments, that the variance at time $t$, $W(t)=E\left[(N(t)-E[N(t)])^{2}\right]$, is super-additive in $t$, and so the $\operatorname{limit}_{\lim } \rightarrow \infty W(t) / t=\sup _{t>0} W(t) / t$ already exists, possibly as an infinite limit. Indeed, one checks

$$
W(t+s)-W(t)-W(s)=2 E[(N(t+s)-N(t)-E[N(s)])(N(t)-E[N(t)])] \geqslant 0 .
$$

The plan will now be to verify that the tagged position $x(t)$ in $d=1$ under the assumptions of Theorem 3 has associated increments. The following is a coupling which essentially says adding more particles to the system slows down or speeds up the tagged particle under conditions (ID $\downarrow$ ) or (ID $\uparrow$ ) respectively.

Lemma 4.1. Suppose in $d=1$ that $p$ is totally asymmetric and nearest-neighbor, namely $p(1)=1$. Then, under Assumption (ID $\downarrow$ ) on $g$, we can couple two copies of the joint process, $\left(x^{1}(t), \xi^{1}(t)\right)$ and $\left(x^{2}(t), \xi^{2}(t)\right)$ where $\xi^{1}(0) \leqslant$ $\xi^{2}(0)$ coordinatewise and $x^{1}(0) \geqslant x^{2}(0)$ so that $\xi^{1}(t) \leqslant \xi^{2}(t)$ and $x^{1}(t) \geqslant x^{2}(t)$ for $t \geqslant 0$.

Similarly, under Assumption (ID $\uparrow$ ), when $\xi^{1}(0) \leqslant \xi^{2}(0)$ and $x^{1}(0) \leqslant x^{2}(0)$, one can couple the systems so that $\xi^{1}(t) \leqslant \xi^{2}(t)$ and $x^{1}(t) \leqslant x^{2}(t)$ for $t \geqslant 0$.

Proof. We prove the statement under (ID $\downarrow$ ) as the proof is analogous with respect to (ID $\uparrow$ ). We now couple so that when an $\xi^{1}$ particle moves, a corresponding $\xi^{2}$ particle also moves to the right, and also when $x^{2}$ would move ahead of $x^{1}$ then $x^{1}$ also moves.

More carefully, at vertex $x \neq x^{1}, x^{2}$, the basic coupling applies - with rate $g\left(\xi_{x}^{1}\right)$ a particle from $x$ in both systems moves; and with rate $g\left(\xi_{x}^{2}\right)-g\left(\xi_{x}^{1}\right)$ a particle from $x$ in system 2 moves.

When $x^{1} \neq x^{2}$, with rate $g\left(\xi_{x^{1}}^{1}\right)\left(\xi_{x^{1}}^{1}-1\right) / \xi_{x^{1}}^{1}$ a non-tagged particle in system 1 and a particle in system 2 move from location $x^{1}$; with rate $g\left(\xi_{x^{1}}^{1}\right) / \xi_{x^{1}}^{1}$ the tagged particle from system 1 and a particle from system 2 at $x^{1}$ move; and with rate $g\left(\xi_{x^{1}}^{2}\right)-g\left(\xi_{x^{1}}^{1}\right)$ a particle in system 2 at $x^{1}$ moves.

With respect to location $x^{2}$, with rate $g\left(\xi_{x^{2}}^{1}\right)\left(\xi_{x^{2}}^{1}-1\right) / \xi_{x^{2}}^{1}$ a particle from system 1 and a non-tagged particle in system 2 move from $x^{2}$; with rate $g\left(\xi_{x^{2}}^{2}\right) / \xi_{x^{2}}^{2}$ the tagged particle in system 2 and a particle in system 1 move from location $x^{2}$; with rate $g\left(\xi_{x^{2}}^{1}\right) / \xi_{x^{2}}^{1}-g\left(\xi_{x^{2}}^{2}\right) / \xi_{x^{2}}^{2}$ a particle in system 1 and a non-tagged particle in system 2 move from $x_{2}$; with the remaining rate

$$
g\left(\xi_{x^{2}}^{2}\right) \frac{\xi_{x^{2}}^{2}-1}{\xi_{x^{2}}^{2}}-g\left(\xi_{x^{2}}^{1}\right) \frac{\xi_{x^{2}}^{1}-1}{\xi_{x^{2}}^{1}}-\frac{g\left(\xi_{x^{2}}^{1}\right)}{\xi_{x^{2}}^{1}}+\frac{g\left(\xi_{x^{2}}^{2}\right)}{\xi_{x^{2}}^{2}}=g\left(\xi_{x_{2}}^{2}\right)-g\left(\xi_{x_{2}}^{1}\right)
$$


a non-tagged particle moves from system 2 at $x^{2}$.

When $x^{1}=x^{2}=x$, with rate $g\left(\xi_{x}^{1}\right)\left(\xi_{x}^{1}-1\right) / \xi_{x}^{1}$ a non-tagged particle from $x$ in both systems moves; with rate $g\left(\xi_{x}^{2}\right) / \xi_{x}^{2}$ both tagged particles move; with rate $g\left(\xi_{x}^{1}\right) / \xi_{x}^{1}-g\left(\xi_{x}^{2}\right) / \xi_{x}^{2}$ the tagged particle in system 1 and a non-tagged particle in system 2 move; with the remaining rate $g\left(\xi_{x}^{2}\right)-g\left(\xi_{x}^{1}\right)$ (cf. (4.1)), a non-tagged particle in system 2 moves.

We omit the generator formulation.

The next lemma owes some intuition to Theorem 2 [9].

Lemma 4.2. In $d=1$, under the assumptions of Theorem 3, the $L^{2}$ process $x(t)$ under $Q_{\alpha}$ has weakly positively associated stationary increments.

Proof. From (1.3), clearly $x(t)=N_{1}(t)$ is an $L^{2}$ process. Also under $Q_{\alpha}, x(t)$ has stationary increments. Consider now the sequence, for increasing $\phi$ and $\psi$,

$$
\begin{aligned}
E_{\alpha}[\phi(x(t+s)-x(t)) \psi(x(t))] & =E_{\alpha}\left[\psi(x(t)) E_{\eta(t)}[\phi(x(s))]\right] \\
& =E_{\alpha}\left[\psi\left(N_{-1}\left(t ; \eta^{*}(\cdot)\right)\right) E_{\eta^{*}(0)}[\phi(x(s))]\right]
\end{aligned}
$$

where in the second step, we reverse time at $t$ with $\eta^{*}(u)=\eta(t-u)$ so that $N_{1}(t ; \eta(\cdot))$, the number of right-shifts up to time $t$ under the forward process, equals $N_{-1}\left(t ; \eta^{*}(\cdot)\right)$, the number of left-shifts up to time $t$ with respect to the reversed process.

As noted in the introduction, the reversed process $\left\{\eta^{*}(u): 0 \leqslant u \leqslant t\right\}$ begun under $Q_{\alpha}$ is also a reference frame zero-range process in equilibrium $Q_{\alpha}$ but with reversed jump probability $p^{*}(\cdot)=p(-\cdot)$ where $p^{*}(-1)=1$. As in Section 2, let $E_{\alpha}^{*}$ and $E_{\eta}^{*}$ denote expectations with respect to the reversed process with initial states $Q_{\alpha}$ and $\eta$ respectively.

Then, after conditioning on time 0 , and noting $N_{-1}(t ; \eta(\cdot))=-x(t)$ under the reversed process,

$$
\begin{aligned}
E_{\alpha}\left[\psi\left(N_{-1}\left(t ; \eta^{*}(\cdot)\right)\right) E_{\eta^{*}(0)}[\phi(x(s))]\right] & =E_{\alpha}^{*}\left[\psi\left(N_{-1}(t ; \eta(\cdot))\right) E_{\eta(0)}[\phi(x(s))]\right] \\
& =\int E_{\eta}^{*}[\psi(-x(t))] E_{\eta}[\phi(x(s))] \mathrm{d} Q_{\alpha}(\eta) .
\end{aligned}
$$

Consider the functions $E_{\eta}^{*}[\psi(-x(t))]$, and $E_{\eta}[\phi(x(s))]$ as functions of $\eta$. Both are coordinatewise in $\eta$ decreasing under (ID $\downarrow$ ) and increasing under (ID $\uparrow$ ) by the coupling in Lemma 4.1. Indeed, the coupling implies that by increasing $\eta$ by one particle, $-x(t)$ under the reversed process and $x(t)$ under the forward process both decrease/increase with assumption (ID $\downarrow) /(\mathrm{ID} \uparrow)$ (recall that the reversed process moves to the left).

With this monotonicity, the associated property follows from the standard FKG inequality for product measures (see Section II.2 [17]).

Proof of Theorem 3. By Lemma 4.2, $x(t)$ has weakly positively associated increments. Hence, by the remark after Theorem 4, the variance $V(t)$ is super-additive in $t \geqslant 0$, and the limit $\sigma^{2}=\lim _{t \rightarrow \infty} V(t) / t$ exists and is in form $\lim _{t \rightarrow \infty} V(t) / t=\sup _{t>0} V(t) / t$. Also, by Theorem 2, $\sup _{t>0} V(t) / t \leqslant C<\infty$. Therefore, the finite-dimensional convergence to a Brownian motion with coefficient $\sigma^{2}$ follows from the Newman-Wright Theorem 4. For the particular evaluation and lower bound on the diffusion coefficient, Theorem 1 straightforwardly applies. The strict lower bound also follows in the case $g(k) \not \equiv c k$ from super-additivity as $V(1)>\alpha / \rho$.

\section{Acknowledgements}

I would like to thank M. Balasz for pointing out Lemma 4.1 would hold under (ID $\uparrow$ ), and O. Zeitouni for simplifying a proof through the remark after Theorem 4. Also, I am grateful to N. Jain, P. Jung, C. Lee, E. Saada, and M. Zerner for useful discussions, and to P. Ferrari and C. Landim for their interest and useful advice. I would also like to acknowledge a FAPESP grant for the nice hospitality on a Summer '04 visit to USPE Sao Paulo and IMPA Rio de Janeiro when part of this work was completed. 


\section{References}

[1] E.D. Andjel, Invariant measures for the zero range process, Ann. Probab. 10 (1982) 525-547.

[2] R. Arratia, The motion of a tagged particle in the simple symmetric exclusion system on $\mathbb{Z}^{1}$, Ann. Probab. 11 (1983) 362-373.

[3] A. De Masi, P. Ferrari, Self-diffusion in one-dimensional lattice gases in the presence of an external field, J. Statist. Phys. 38 (1985) $603-613$.

[4] S. Ethier, T. Kurtz, Markov Processes: Characterization and Convergence, J. Wiley and Sons, New York, 1986.

[5] P. Ferrari, Limit theorems for tagged particles, Markov Processes Related Fields 2 (1996) 17-40.

[6] S. Grosskinsky, G.M. Schütz, H. Spohn, Condensation in the zero range process: stationary and dynamical properties, J. Statist. Phys. 113 (2003) 389-410.

[7] M. Jara, C. Landim, Nonequilibrium central limit theorem for a tagged particle in symmetric simple exclusion, Ann. Inst. H. Poincaré Probab. Statist., in press.

[8] I. Jeon, P. March, B. Pittel, Size of the largest cluster under zero-range invariant measures, Ann. Probab. 28 (2000) $1162-1194$.

[9] C. Kipnis, Central limit theorems for infinite series of queues and applications to simple exclusion, Ann. Probab. 14 (1986) $397-408$.

[10] C. Kipnis, C. Landim, Scaling Limits of Interacting Particle Systems, Grundlehren der Mathematischen Wissenschaften, vol. 320, SpringerVerlag, Berlin, 1999.

[11] C. Kipnis, S.R.S. Varadhan, Central limit theorem for additive functionals of reversible Markov processes, Comm. Math. Phys. 104 (1986) $1-19$.

[12] C. Landim, S. Olla, S. Volchan, Driven tracer particle in one dimensional symmetric simple exclusion process, Comm. Math. Phys. 192 (1998) 287-307.

[13] C. Landim, S. Sethuraman, S.R.S. Varadhan, Spectral gap for zero range dynamics, Ann. Probab. 24 (1996) 1871-1902.

[14] C. Landim, H.T. Yau, Fluctuation-dissipation equation of asymmetric simple exclusion processes, Probab. Theory Related Fields 108 (1997) 321-356.

[15] C. Landim, S. Volchan, Equilibrium fluctuations for a driven tracer particle dynamics, Stochastic Process Appl. 85 (2000) $139-158$.

[16] T.M. Liggett, An infinite particle system with zero range interactions, Ann. Probab. 1 (1973) 240-253.

[17] T.M. Liggett, Interacting Particle Systems, Grundlehren der Mathematischen Wissenschaften, vol. 276, Springer-Verlag, New York, 1985.

[18] T.M. Liggett, Stochastic Interacting Systems: Contact, Voter and Exclusion Processes, Grundlehren der Mathematischen Wissenschaften, vol. 324, Springer-Verlag, Berlin, 1999.

[19] B. Morris, Spectral gap for the zero range process with constant rate, preprint, math.PR/0405161, 2004.

[20] C.M. Newman, A General Central Limit Theorem for FKG systems, Comm. Math. Phys. 91 (1983) 75-80.

[21] C.M. Newman, Asymptotic independence and limit theorems for positively and negatively dependent random variables, in: Inequalities in Statistics and Probability, in: IMS Lecture Notes Monograph Series, vol. 5, 1984, pp. 127-140.

[22] S. Olla, Homogenization of diffusion processes in random fields, École Polytechnique Lecture Notes, 1994.

[23] S.C. Port, C.J. Stone, Infinite particle systems, Trans. Amer. Math. Soc. 178 (1973) 307-340.

[24] J. Quastel, F. Rezakhanlou, S.R.S. Varadhan, Large deviations for the symmetric simple exclusion process in dimensions $d \geqslant 3$, Probab. Theory Related Fields 113 (1999) 1-84.

[25] F. Rezakhanlou, Propagation of chaos for symmetric simple exclusions, Comm. Pure Appl. Math. 47 (1994) $943-957$.

[26] F. Rezakhanlou, Evolution of tagged particles in non-reversible particle systems, Comm. Math. Phys. 165 (1994) 1-32.

[27] H. Rost, M.E. Vares, Hydrodynamics of a one dimensional nearest neighbor model, Contemp. Math. 41 (1985) 329-342.

[28] E. Saada, Processus de zero range et d'exclusion simple avec particule marquee, Ph.D. Thesis, 3rd Cycle, L'Universite Pierre et Marie Curie Paris 6, 1985.

[29] E. Saada, A limit theorem for the position of a tagged particle in a simple exclusion process, Ann. Probab. 15 (1) (1987) $375-381$.

[30] E. Saada, Processus de zero-range avec particule marquee, Ann. Inst. H. Poincaré 26 (1990) 5-17.

[31] T. Seppäläinen, Coupling the totally asymmetric simple exclusion process with a moving interface, Markov Process Related Fields 4 (1998) 593-628.

[32] S. Sethuraman, On extremal measures for conservative particle systems, Ann. Inst. H. Poincaré Probab. Statist. 37 (2001) $139-154$.

[33] S. Sethuraman, S.R.S. Varadhan, H.T. Yau, Diffusive limit of a tagged particle in asymmetric simple exclusion processes, Comm. Pure Appl. Math. 53 (2000) 972-1006.

[34] S. Sethuraman, L. Xu, A central limit theorem for reversible exclusion and zero-range particle systems, Ann. Probab. 24 (1996) $1842-1870$.

[35] F. Spitzer, Interaction of Markov processes, Adv. Math. 5 (1970) 246-290.

[36] H. Spohn, Large Scale Dynamics of Interacting Particles, Springer-Verlag, Berlin, 1991.

[37] S.R.S. Varadhan, Self-diffusion of a tagged particle in equilibrium for asymmetric mean zero random walk with simple exclusion, Ann. Inst. H. Poincaré Probab. Statist. 31 (1995) 273-285. 